\title{
Monodromy at Infinity and Fourier Transform
}

By

\author{
Claude SABBAH*
}

\begin{abstract}
Let $f: U \rightarrow \mathbf{C}$ be a regular function on a smooth quasi-projective variety $U$. We compute the limit mixed Hodge structure (when $t \rightarrow \infty$ ) of the cohomology of the fibers $f=t$ in terms of the Fourier transform of the Gauss-Manin system associated to $f$.
\end{abstract}

\section{Introduction}

Let $f: U \rightarrow \mathbf{C}$ be a regular function on a smooth quasi-projective variety $U$. For $t \in \mathbf{C}$, the cohomology spaces $H^{\bullet}\left(f^{-1}(t), \mathbf{Q}\right)$ underly a natural mixed Hodge structure (cf. [6] for $t$ generic and [7] if $f^{-1}(t)$ is singular). Steenbrink and Zucker [26] (see also [8]) have constructed a limit mixed Hodge structure when $t \rightarrow \infty$.

This mixed Hodge structure can also be obtained [24] by compactifying $f$ as a map $F: \mathscr{X} \rightarrow \mathbf{P}^{1}$ with $\mathscr{X}$ smooth, and by constructing a mixed Hodge module structure on the nearby cycles at $t=\infty$ of the sheaf $\boldsymbol{R} \kappa_{*} \mathbf{Q}_{U}$, if $\kappa: U \hookrightarrow \mathscr{X}$ denotes the inclusion: one obtains the Steenbrink-Zucker limit by taking the global de Rham complex of this mixed Hodge module on $F^{-1}(\infty)$.

This paper proposes to recover this limit mixed Hodge structure using Fourier transform techniques. The main object is the $\mathscr{D}_{x}[\tau]\left\langle\partial_{\tau}\right\rangle$-module $\kappa_{+} \mathscr{E}^{-\tau f}$, where $\mathscr{E}^{-\tau f}$ is $\mathcal{O}_{U}[\tau]$ equipped with the natural $\mathscr{D}_{x}[\tau]\left\langle\partial_{\tau}\right\rangle$-action twisted by $e^{-\tau f}$, and $\kappa$ still denotes the inclusion $U \times \operatorname{Spec} \mathbf{C}[\tau] \subseteq \mathscr{X} \times \operatorname{Spec} \mathbf{C}[\tau]$. This module is holonomic but not regular in general, so does not enter in the frame of mixed Hodge module theory. However, as $\kappa_{+} \mathcal{O}_{U}$ is regular on $\mathscr{X}$ (Grothendieck comparison theorem), $\kappa_{+} \mathscr{E}^{-\tau f}$ is regular along $\tau=0$, so one may compute the vanishing cycles along $\tau=0$ of its de Rham complex using the Malgrange-Kashiwara filtration and apply a procedure analogous

Communicated by M. Kashiwara, September 17, 1996.

1991 Math. Subject Classification(s): 14D07, 14D05, 32G20, 32S40.

* URA 169 du C.N.R.S., Centre de Mathématiques, Ecole Polytechnique, F-91128 Palaiseau cedex, France (sabbah@math.polytechnique.fr). 
to the one of [23].

In $\S 1$ some known facts concerning Fourier transform with parameters of sheaves are recalled. In $\S 2$ Fourier transform with parameters of holonomic $\mathscr{D}$-modules is introduced and a proof of a comparison theorem between both kinds of Fourier transforms is given, in the case of regular holonomic modules and their de Rham complex (such a theorem is also proved in [5] in a more general situation and the case without parameters is treated in [14]).

The main result of $\S 3$ is that one may apply the theory of Malgrange-Kashiwara filtration to Fourier transforms of regular holonomic $\mathscr{D}$-modules to compute the nearby and vanishing cycles along $\tau=0$.

Section 4 is dedicated to Hodge theory of vanishing cycles at $\tau=0$ of the Fourier transform relative to $f$ of $\kappa_{+} \mathcal{O}_{U}$ (and also the nearby cycles), namely the $\mathscr{D}_{\mathscr{P}}$-module $\phi_{\tau}^{\text {mod }} \kappa_{+} \mathscr{E}^{-\tau f}$. A filtration naturally defined in terms of a natural filtration on $\kappa_{+} \mathscr{E}^{-\tau f}$ is put on this object (following the method developed by M. Saito [23] using the Malgrange-Kashiwara filtration) and it is shown that the isomorphisms of $\S \S 1,2$ identify this filtered module with the mixed Hodge module of nearby cycles at infinity ${ }^{p} \psi_{1 / F}\left(\mathbb{R} \kappa_{*} Q_{U}\right)$ defined by M. Saito.

In $\S 5$ applications are given to the computation of the limit mixed Hodge structure of Steenbrink and Zucker in terms of the Fourier transform of the Gauss-Manin system of $f$.

The reader is referred to the appendix for the notation which is not defined in the main course, in particular for the conventions used concering perverse functors.

I thank B. Malgrange for simplifying and clarifying some statements and proofs of a previous version of the paper.

\section{$\S 1$. Sheaf-theoretical Fourier Transform}

We review here some variants of well-known facts concerning the Fourier transform of non-necessarily homogeneous sheaves (see [14] and the references given there, see also e.g. [11, chap. III] for the Fourier-Sato transform of homogeneous sheaves on a vector space and $[4,12]$ for the $l$-adic analogue).

Some notation. In the following, $\mathbb{A}^{1}$ will denote the affine line with affine coordinate $t$ and $\check{\mathbf{A}}^{1}$ the affine line with coordinate $\tau$. In this section they will come equipped with their analytic topology. Let $\mathbb{P}^{1}=\mathbb{A}^{1} \cup\{\infty\}$ be the 
projective line and let $\varepsilon: \widetilde{\mathbf{P}}^{1} \rightarrow \mathbb{P}^{1}$ be the real oriented blowing-up of $\mathbf{P}^{1}$ at $\infty$. We have $\widetilde{\mathbf{P}}^{1}=\mathbf{A}^{1} \cup S^{1}$ and we denote $\theta=\arg t$ the coordinate on $S^{1}$. Consider the following diagram

$$
\begin{array}{cccc}
\mathbf{A}^{1} \times \check{\mathbf{A}}^{1} & \stackrel{\tilde{k}}{G} & \tilde{\mathbf{P}}^{1} \times \check{\mathbf{A}}^{1} & \stackrel{\check{q}}{\rightarrow} \check{\mathbf{A}}^{1} \\
\| & & \downarrow \varepsilon \times \mathrm{Id} & \| \\
\mathbf{A}^{1} \times \check{\mathbf{A}}^{1} & \stackrel{k}{G} & \mathbf{P}^{1} \times \check{\mathbf{A}}^{1} & \stackrel{q}{\rightarrow} \\
\downarrow & \check{\mathbf{A}}^{1} \\
\downarrow p & & \downarrow p \\
\mathbf{A}^{1} & \stackrel{k}{G} & \mathbf{P}^{1}
\end{array}
$$

Denote $i:\{\infty\} \hookrightarrow \mathbb{P}^{1}$ (resp. $\tilde{l}: S^{1} \hookrightarrow \widetilde{\mathbb{P}}^{1}$ ) the complementary inclusion of $k$ (resp. $\tilde{k}$ ); denote similarly $\check{l}:\{0\} \hookrightarrow \check{\mathbf{A}}^{1}$ and $\check{k}: \check{\mathbf{A}}^{1}-\{0\} \hookrightarrow \check{\mathbf{A}}$ the complementary inclusions in the space $\check{\mathbf{A}}^{1}$. We keep the same notation after taking the product with $\check{\mathbf{A}}^{1}$ (for $i$ and $\tilde{l}$ ) or with $\mathbf{A}^{1}$ (for $\check{l}, \check{k}$ ).

Denote $L^{\prime+}$ the closed set of $S^{1} \times \check{\mathrm{A}}^{1}$ defined by $\operatorname{Re}\left(e^{i \theta} \tau\right) \geq 0$ and let $L^{-}$ be its complement in $\widetilde{\mathbf{P}}^{1} \times \check{\mathbf{A}}^{1}\left(L^{-}\right.$contains $\mathbf{A}^{1} \times \check{\mathbf{A}}^{1}$ as an open set). Notice that the fibre of $L^{\prime+}$ over $\tau=0$ is equal to $S^{1}$, so the corresponding fibre of $L^{-}$is empty.

Let $Q_{L^{\prime}+}$ be the constant sheaf on the closed set $L^{\prime+}$ extended by 0 on $\widetilde{\mathbf{P}}^{1} \times \check{\mathbf{A}}^{1}$.

Let $Q_{L^{-}}$be the constant sheaf on the open set $L^{-}$extended by 0 on $\widetilde{\mathbf{P}}^{1} \times \check{\mathbf{A}}^{1}$. In particular, the restriction of $\mathbf{Q}_{L^{-}}$to $\widetilde{\mathbf{P}}^{1} \times\{0\}$ is equal to the extension by 0 of the constant sheaf $\mathbf{Q}_{\mathbf{A}^{1}}$.

We hence have a triangle of $D\left(\mathbf{Q}_{\widetilde{\mathbf{P}}^{1} \times \check{\mathbf{A}}^{1}}\right)$

$$
\mathbf{Q}_{L^{-}} \rightarrow \mathbf{Q}_{\tilde{\mathbf{P}}^{1} \times \check{\mathbf{A}}^{1}} \rightarrow \mathbb{Q}_{L^{j^{+}}} \stackrel{+1}{\rightarrow}
$$

Fourier transform. Let $\mathscr{X}$ be an analytic manifold. We use the same notation for the diagram obtained from the previous one after taking the product with $\mathscr{X}$. We will use the two following functors of triangulated categories

$$
\begin{aligned}
& \mathfrak{F}_{\mathscr{X}}: D^{+}\left(\mathbf{Q}_{\mathscr{X} \times \mathbf{A}^{1}}\right) \rightarrow D^{+}\left(\mathbf{Q}_{\mathscr{X} \times \check{\mathbf{A}}^{1}}\right), \quad \mathfrak{F}_{\mathscr{X}}(\mathscr{F})=\boldsymbol{R} \check{q}_{*}\left(\boldsymbol{R} \tilde{k}_{*} p^{-1} \mathscr{F}[1] \otimes \mathbf{Q}_{\mathscr{X} \times L^{-}}\right) \\
& \text {(1.2) } \mathfrak{F}_{\mathscr{X}}^{\mathrm{ir}}: D^{+}\left(\mathbf{Q}_{\mathscr{X} \times \mathbf{A}^{1}}\right) \rightarrow D^{+}\left(\mathbf{Q}_{\mathscr{X} \times \check{\mathbf{A}}^{1}}\right), \quad \mathfrak{F}_{\mathscr{X}}^{\mathrm{ir}}(\mathscr{F})=\boldsymbol{R} \check{q}_{*}\left(\boldsymbol{R} \tilde{k}_{*} p^{-1} \mathscr{F} \otimes \mathbf{Q}_{\mathscr{X} \times L^{\prime+}}\right)
\end{aligned}
$$

which come in a triangle of $D^{+}\left(\mathbf{Q}_{\mathscr{X} \times \check{\mathbf{A}}^{1}}\right)$

$$
\check{\pi}^{-1} \mathbb{R} \pi_{*} \mathscr{F} \rightarrow \mathscr{F}_{\mathscr{X}}^{\mathrm{ir}}(\mathscr{F}) \rightarrow \mathfrak{F}_{\mathscr{X}}(\mathscr{F}) \stackrel{+1}{\rightarrow}
$$


where $\pi$ (resp. $\check{\pi})$ denotes the projection of $\mathscr{X} \times \mathbb{A}^{1}\left(\right.$ resp. $\left.\mathscr{X} \times \check{\mathbb{A}}^{1}\right)$ onto $\mathscr{X}$.

Remark. Denote $\alpha: \mathbb{A}^{1} \times \check{\mathbb{A}}^{1} \varsigma L^{-}$the open inclusion and $\check{q}^{-}: L^{-} \rightarrow \check{\mathbb{A}}^{1}$ the restriction of $\check{q}$ to $L^{-}$, and keep the same notation after taking the product by $\mathscr{X}$. The formula for $\mathfrak{F}_{\mathscr{X}}$ can also be written

$$
\mathfrak{F}_{\mathscr{T}}(\mathscr{F})=\boldsymbol{R} \check{q}_{1}^{-} \boldsymbol{R} \alpha_{*} p^{-1} \mathscr{F}[1] \text {. }
$$

1.5. Fourier transform relative to a meromorphic function. Let $F: \mathscr{X} \rightarrow \mathbb{P}^{1}$ be a meromorphic function on $\mathscr{X}$. Consider the following diagram of maps (and the same one after taking the product by $\check{\mathrm{A}}^{1}$ ):

$$
\begin{array}{cccccc}
X & \stackrel{k_{F}}{\leftrightarrows} & \tilde{X} & \stackrel{\tilde{F}}{\rightarrow} & \tilde{\mathbf{P}}^{1} \\
\| & \varepsilon \downarrow & \square & \downarrow \varepsilon \\
F^{-1}\left(\mathbf{A}^{1}\right) \stackrel{\text { def }}{=} X & \stackrel{k_{F}}{\leftrightarrows} & \mathscr{X} & \stackrel{F}{\rightarrow} & \mathbb{P}^{1}
\end{array}
$$

where $\tilde{X}$ is the fibre product $\mathscr{X} \times{ }_{\mathbb{P}^{1}} \tilde{\mathbf{P}}^{1}$ and $X$ denotes the restriction of $\mathscr{X}$ over $\mathbf{A}^{1}$.

Let $L_{F}^{-} \subset \tilde{\mathscr{X}} \times \check{\mathbb{A}}^{1}$ be the inverse image of $L^{-} \subset \widetilde{\mathbb{P}}^{1} \times \check{\mathbb{A}}^{1}$ by $\tilde{F} \times I d$. The Fourier transform relative to $F$ is the functor

$$
\mathfrak{F}_{F}: D^{+}\left(\mathbf{Q}_{X}\right) \rightarrow D^{+}\left(\mathbf{Q}_{\mathscr{X} \times \check{\mathbb{A}}^{1}}\right), \quad \mathfrak{F}_{F}(\mathscr{F})=\boldsymbol{R} \varepsilon_{*}\left(\boldsymbol{R}{\tilde{k_{F} *}} p^{-1} \mathscr{F}[1] \otimes \mathbf{Q}_{L_{\bar{F}}}\right) .
$$

Considering the following diagram

$$
\begin{aligned}
& X \times \check{\mathbf{A}}^{1} \quad \stackrel{\alpha_{F}}{\leftrightarrows} \quad L_{F}^{-} \quad \stackrel{\beta_{F}}{\leftrightarrows} \quad \tilde{\mathscr{X}} \times \check{\mathbf{A}}^{1} \quad \stackrel{\varepsilon}{\rightarrow} \mathscr{X} \times \check{\mathbf{A}}^{1} \\
& i_{F}^{\prime} \downarrow \quad \downarrow \quad \square \quad \downarrow \quad \| \\
& \mathscr{X} \times \mathbf{A}^{1} \times \check{\mathbf{A}}^{1} \stackrel{\alpha}{\hookrightarrow} \mathscr{X} \times L^{-} \stackrel{\beta}{\hookrightarrow} \mathscr{X} \times \tilde{\mathbb{P}}^{1} \times \check{\mathbf{A}}^{1} \stackrel{\breve{q}}{\rightarrow} \mathscr{X} \times \check{\mathbb{A}}^{1}
\end{aligned}
$$

where $i_{F}^{\prime}$ is the composition of the graph embedding $X \varsigma X \times \mathbb{A}^{1}\left(\times \check{\mathbb{A}}^{1}\right)$ and $k_{F}$, one has $\mathfrak{F}_{F}(\mathscr{F})=\boldsymbol{R}\left(\varepsilon \circ \beta_{F}\right)_{!} \boldsymbol{R} \alpha_{F *} p^{-1} \mathscr{F}[1]$ and one shows that

$$
\mathfrak{F}_{F}=\mathfrak{F}_{\mathscr{X}} \circ \boldsymbol{R} i_{F *}^{\prime}
$$

We also put $\mathfrak{F}_{F}^{\text {id }} \stackrel{\text { def }}{=} \mathfrak{F}_{\mathscr{X}}^{\text {ir }} \circ R i_{F *}^{\prime}$ so that the triangle $(1.3)$ becomes

$$
\check{\pi}^{-1} R k_{F *} \mathscr{F} \rightarrow \mathscr{\mho}_{F}^{\mathrm{ir}}(\mathscr{F}) \rightarrow \mathfrak{\mho}_{F}(\mathscr{F}) \stackrel{+1}{\rightarrow}
$$


Let $j: U \hookrightarrow X$ be a Zariski open set (in the analytic sense). Put $\kappa \stackrel{\text { def }}{=} k_{F} \circ j$ and $\tilde{\kappa}=\tilde{k}_{F} \circ j$. One has

$$
\mathfrak{F}_{F}\left(\boldsymbol{R} j_{*}^{p} \mathbf{Q}_{U}\right)=\boldsymbol{R} \varepsilon_{*}\left(\boldsymbol{R} \tilde{\kappa}_{*}^{p} \mathbf{Q}_{U \times \check{\mathbf{A}}^{1}} \otimes \mathbf{Q}_{L_{\bar{F}}}\right)=\boldsymbol{R}\left(\varepsilon \circ \beta_{F}\right)_{l} \boldsymbol{R}\left(\alpha_{F} \circ j\right)_{*}^{p} \mathbf{Q}_{U \times \check{\mathbf{A}}^{1}} .
$$

1.9. Nearby and vanishing cycles. We will consider the functor of vanishing cycles at $t=\infty$ shifted by -1 (see $\S$ A.2 for the conventions made in this paper) which we denote ${ }^{p} \psi_{1 / t}$. This is a functor from the category $D_{c}^{b}\left(\mathbf{Q}_{x \times \check{\mathbf{A}}^{1}}\right)$ (bounded complexes with constructible cohomology) to the category $D_{c}^{b}\left(\mathbf{Q}\left[T, T^{-1}\right]_{x}\right)$ (bounded complexs with constructible cohomology as sheaves of $\mathbf{Q}$-vector spaces and $\mathbf{Q}\left[T, T^{-1}\right]$-modules). Here, $T$ denotes the monodromy along a positively oriented circle in the variable $1 / t$. We will also denote $T_{\infty}=T^{-1}$ the monodromy along a positively oriented circle of big radius in the variable $t$.

Denote $\mathscr{L}$ the local system on $\mathbf{A}^{1 *}=\mathbf{A}^{1}-\{0\}$ with fibre $\mathbf{Q}\left[T, T^{-1}\right]$ and monodromy (in the first sense) given by the multiplication by $T$. We keep the same notation for its inverse image on a product space like $\mathscr{X} \times \mathbf{A}^{1 *}$. For an object $\mathscr{F}$ of $D_{c}^{b}\left(\mathbf{Q}_{X \times \mathbf{A}^{1}}\right)$ we have (see e.g. [4])

$$
{ }^{p} \psi_{1 / t} \mathscr{F}=i^{-1} R k_{*}(\mathscr{F} \otimes \mathscr{L}) .
$$

Identifying the constant sheaf on $\mathbf{A}^{1 *}$ with $\mathscr{L} /(T-1) \mathscr{L}$, the natural morphism

$$
i^{-1} \boldsymbol{R} k_{*} \mathscr{F}[-1] \rightarrow{ }^{p} \psi_{1 / t^{\mathscr{F}}}
$$

is obtained from the morphism of complexes

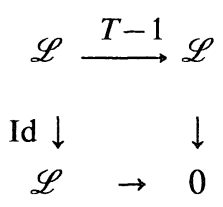

We will also consider the functors ${ }^{p} \psi_{\tau}$ and ${ }^{p} \phi_{\tau}$ of nearby and vanishing cycles at $\tau=0$, as functors from $D_{c}^{b}\left(\mathbf{Q}_{\mathscr{X} \times \check{\mathbf{A}}^{1}}\right)$ to $D_{c}^{b}\left(\mathbf{Q}\left[\check{T}, \check{T}^{-1}\right]_{\mathscr{X}}\right)$. They are defined using the local system $\breve{\mathscr{L}}$ on $\check{\mathrm{A}}^{1}-\{0\}$ (see e.g. $[4,11]$ ).

(1.10) Theorem. Let $\mathscr{X}$ be a smooth complex analytic manifold.

1. The functors $\mathfrak{F}_{\mathscr{X}}$ and $\mathfrak{F}_{\mathscr{X}}^{\mathrm{ir}}$ send $D_{c}^{b}\left(\mathbf{Q}_{\mathscr{X} \times \mathbf{A}^{1}}\right)$ in $D_{c}^{b}\left(\mathbf{Q}_{\mathscr{X} \times \check{\mathbf{A}}^{1}}\right)$ and induce exact 
functors from the abelian category $\operatorname{Perv}\left(\mathbb{Q}_{\mathscr{X} \times \mathbf{A}^{1}}\right)$ to $\operatorname{Perv}\left(\mathbb{Q}_{\mathscr{X} \times \overleftarrow{\mathbb{A}}^{1}}\right)$.

2. There are isomorphisms between

(a) the functor ${ }^{p} \psi_{1 / t}$ of nearby cycles along $t=\infty$ and the composed functor ${ }^{p} \psi_{\tau} \circ \mathfrak{F}_{\mathscr{X}}^{\mathrm{ir}}$ from $D_{c}^{b}\left(\mathbb{Q}_{\mathscr{X} \times \mathbf{A}^{1}}\right)$ to $D_{c}^{b}\left(\mathbb{Q}\left[T, T^{-1}\right]_{\mathscr{X}}\right)$ after the identification $\check{T}=T$,

(b) the functors $i^{-1} \mathbb{R} k_{*}$ and $\check{i}^{-1} \mathscr{\mho}_{\mathscr{X}}^{\mathrm{ir}}$ from $D_{c}^{b}\left(\mathbb{Q}_{\mathscr{X} \times \mathbf{A}^{1}}\right)$ to $D_{c}^{b}\left(\mathbb{Q}_{\mathscr{X}}\right)$, which are compatible with the canonical morphisms of functors $i^{-1} R k_{*} \mathscr{F}[-1] \rightarrow^{p} \psi_{1 / t} \mathscr{F}$ and $\check{l}^{-1} \mathfrak{\mho}_{x}^{\mathrm{ir}}(\mathscr{F})[-1] \rightarrow^{p} \psi_{\tau} \circ \mathscr{F}_{\mathscr{X}}^{\mathrm{ir}}(\mathscr{F})$.

3. The variation var: ${ }^{p} \phi_{\tau} \circ \mathfrak{F}_{\mathscr{X}}^{\mathrm{ir}} \rightarrow{ }^{p} \psi_{\tau} \circ \mathfrak{F}_{\mathscr{X}}^{\mathrm{ir}}$ is an isomorphism.

Let now $F: \mathscr{X} \rightarrow \mathbb{P}^{1}$ as in $\S 1.5$ and let $\mathscr{F}$ be in $D_{c}^{b}\left(Q_{X}\right)$. Property (3) above is equivalent to $\iota^{\prime} \mathfrak{F}_{\mathscr{T}}^{\mathrm{ir}}(\mathscr{F})=0$, so from (1.7) we get

$$
\check{l}^{!} \mathfrak{F}_{F}(\mathscr{F})[1] \simeq \check{l}^{!} \check{\pi}^{-1} \mathbb{R} k_{F} *^{\mathscr{F}}[2]=\mathbb{R} k_{F} \mathscr{F}^{\mathscr{F}}
$$

as $\check{\pi}$ is smooth. In particular, when $\mathscr{F}$ is in $\operatorname{Perv}\left(\mathbb{Q}_{X}\right)$, the object $\check{l}^{\prime} \mathfrak{F}_{F}(\mathscr{F})[1]$ is in $\operatorname{Perv}\left(\mathbf{Q}_{x}\right)$. In such a situation we have two exact sequences in $\operatorname{Perv}\left(\mathbb{Q}_{\mathscr{X}}\right)$

$$
\begin{gathered}
0 \rightarrow{ }^{p} \phi_{\tau, 1} \circ \mathscr{F}_{F}(\mathscr{F}) \stackrel{\operatorname{var}}{\rightarrow}{ }^{p} \psi_{\tau, 1} \circ \mathscr{F}_{F}(\mathscr{F}) \rightarrow \iota^{\prime} \mathscr{F}_{F}(\mathscr{F})[1] \rightarrow 0 \\
0 \rightarrow{ }^{p} \psi_{1 / F, 1} \mathbb{R} k_{F *} \mathscr{F} \rightarrow \Xi_{F} \mathscr{F} \rightarrow \mathbb{R} k_{F *} \mathscr{F} \rightarrow 0
\end{gathered}
$$

where $\Xi_{F}$ is the Beilinson functor (see e.g. $[24, \S(2 \mathrm{e})]$ and below). $\left.\left.T^{-1}\right]_{X}\right)$

(1.13) Corollary. There exists a functorial isomorphism of $D_{c}^{b}(\mathbb{Q}[T$,

$$
{ }^{p} \psi_{1 / F} \mathbb{R} k_{F *} \mathscr{F} \simeq{ }^{p} \phi_{\tau} \widetilde{F}_{F}(\mathscr{F}) \text { for } \mathscr{F} \text { in } D_{c}^{b}\left(\mathbb{Q}_{X}\right)
$$

and for the eigenvalue 1 of monodromy and for $\mathscr{F} \in \operatorname{Perv}\left(\mathbb{Q}_{X}\right)$ it is part of a functorial isomorphism between the two exact sequences above.

Proof. For the first part, according to the theorem, it is enough to prove that the morphism (1.3) induces an isomorphism ${ }^{p} \phi_{\tau} \circ \mathfrak{F}_{X}^{\text {ir }} \rightarrow{ }^{p} \phi_{\tau} \circ \mathfrak{F}_{X}$. This follows from ${ }^{p} \phi_{\tau}\left(\check{\pi}^{-1} \mathscr{G}\right)=0$ for $\mathscr{G}$ in $D_{c}^{b}\left(\mathbf{Q}_{x}\right)$.

Consider now the cartesian square 


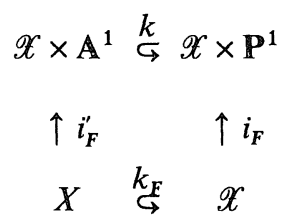

where $i_{F}$ is the graph embedding relative to $F$ and $i_{F}^{\prime}$ as in $\S 1.5$. Let $j_{F}$ be the inclusion complementary to $i_{F}$ and, for $\mathscr{F}$ in $D_{c}^{b}\left(\mathbf{Q}_{X}\right)$, put $\mathscr{G}=$ $k^{-1} j_{F} j_{F}^{-1} \pi^{-1} R k_{F *} \mathscr{F}[1]$ with $\pi: \mathscr{X} \times \mathbb{P}^{1} \rightarrow \mathscr{X}$. We have a triangle of $D_{c}^{b}\left(\mathbf{Q}_{\mathscr{X} \times \mathbf{A}^{1}}\right)$

$$
\boldsymbol{R} i_{F}^{\prime} \mathscr{F} \rightarrow \mathscr{G} \rightarrow \pi^{-1} \boldsymbol{R} k_{F} \mathscr{F}[1] \stackrel{+1}{\longrightarrow}
$$

with now $\pi: \mathscr{X} \times \mathbb{A}^{1} \rightarrow \mathscr{X}$. By definition, $\Xi_{F} \mathscr{G}={ }^{p} \psi_{1 / t, 1} \mathscr{G}$, so from the theorem we get

$$
(1.12)={ }^{p} \psi_{1 / t, 1}(1.14)={ }^{p} \psi_{\tau, 1} \circ \mathfrak{F}_{x}^{\mathrm{ir}}(1.14)
$$

We now use the following commutative diagram, where the morphisms come from (1.7) or (1.14):

$$
\begin{array}{ccc}
{ }^{p} \phi_{\tau, 1} \circ \mathfrak{F}_{F}(\mathscr{F}) & \stackrel{\operatorname{var}}{\rightarrow} & p \psi_{\tau, 1} \circ \mathfrak{F}_{F}(\mathscr{F}) \\
\uparrow l & \downarrow \\
{ }^{p} \phi_{\tau, 1} \circ \mathfrak{F}_{F}^{\mathrm{ir}}(\mathscr{F}) & { }^{p} \psi_{\tau, 1} \circ \mathfrak{F}_{\mathscr{X}}(\mathscr{G}) \\
\operatorname{var} \downarrow l & \uparrow \\
{ }^{p} \psi_{\tau, 1} \circ \mathfrak{F}_{F}^{\mathrm{ir}}(\mathscr{F}) & \rightarrow & { }^{p} \psi_{\tau, 1} \circ \mathfrak{F}_{\mathscr{X}}^{\mathrm{ir}}(\mathscr{G})
\end{array}
$$

The right upper vertical arrow is an isomorphism because for $\mathscr{F}^{\prime}$ in $D_{c}^{b}\left(\mathbf{Q}_{\mathscr{X}}\right)$ the Fourier transform $\mathfrak{F}_{\mathscr{X}}\left(\pi^{-1} \mathscr{F}^{\prime}\right)$ is supported on $\mathscr{X} \times\{0\} \subset \mathscr{X} \times \check{\mathbf{A}}^{1}$ and the lower one is an isomorphism because $\boldsymbol{R} \pi_{*} \mathscr{G}=0$. Both facts are left to the reader.

Proof of Theorem 1.10. It is enough to prove the first part of the theorem over the field $\mathbf{C}$. It is then a consequence of the comparison Theorem 2.2 of the next section and of the Riemann-Hilbert correspondence $[9,15]$ (see also [3]). The third part follows from

(1.15) Lemma. We have $i^{-1} \mathfrak{F}_{\mathscr{X}}^{\mathrm{ir}}(\mathscr{F})=i^{-1} \mathbb{R} k_{*} \mathscr{F}$ and $\check{l}^{!} \mathfrak{F}_{\mathscr{X}}^{\mathrm{ir}}(\mathscr{F})=0$ (which is 
equivalent to $\left.\mathfrak{F}_{\mathscr{X}}^{\mathrm{ir}}(\mathscr{F})=\boldsymbol{R} \check{k}_{*} \check{k}^{-1} \mathfrak{F}_{\mathscr{X}}^{\mathrm{ir}}(\mathscr{F})\right)$.

Proof. Notice first that we have

$$
\boldsymbol{R} k_{*} p^{-1} \mathscr{F}=p^{-1} \boldsymbol{R} k_{*} \mathscr{F} \text { and } \boldsymbol{R} \tilde{\boldsymbol{k}_{*}} p^{-1} \mathscr{F}=p^{-1} \boldsymbol{R} \tilde{\boldsymbol{k}_{*}} \mathscr{F}
$$

indeed, these equalities are obtained from [11, Prop. 2.6.7] by duality, since $p$ is smooth. The first point is then easy, denoting $\pi$ (resp. $\tilde{\pi}$ ) the projection of $\mathscr{X} \times \mathbf{P}^{1}\left(\right.$ resp. $\left.\mathscr{X} \times \widetilde{\mathbf{P}}^{1}\right)$ on $\mathscr{X}$ :

$$
\begin{aligned}
\check{l}^{-1} \boldsymbol{R} \check{q}_{*}\left(\boldsymbol{R} \tilde{k}_{*} p^{-1} \mathscr{F} \otimes\right. & \left.\mathbf{Q}_{\mathscr{X} \times L^{\prime+}}\right) \\
& =\boldsymbol{R} \tilde{\pi}_{*} \check{l}^{-1}\left(\boldsymbol{R} \tilde{k}_{*} p^{-1} \mathscr{F} \otimes \mathbf{Q}_{\mathscr{X} \times L^{\prime+}}\right) \quad(\check{q} \text { proper) } \\
& \left.=\boldsymbol{R} \tilde{\pi}_{*} \check{l}^{-1} \tilde{l}^{-1} \boldsymbol{R} \tilde{k}_{*} p^{-1} \mathscr{F} \text { (the fibre of } L^{\prime+} \text { at } \tau=0 \text { is } S^{1}\right) \\
& =\boldsymbol{R} \tilde{\pi}_{*} \tilde{I}^{-1} \boldsymbol{R} \tilde{k}_{*} \mathscr{F} \text { (previous remark) } \\
& =\boldsymbol{R} \pi_{*} i^{-1} \boldsymbol{R} k_{*} \mathscr{F}=i^{-1} \boldsymbol{R} k_{*} \mathscr{F} .
\end{aligned}
$$

To prove the second point, it will be convenient to consider also the real blowing up of $\check{\mathbf{A}}^{1}$ at $\tau=0$. Put $\widetilde{\mathbf{A}}^{1}=\check{S}^{1} \times[0,+\infty[$ and let $\check{\theta}$ be the coordinate on $\check{S}^{1}$. We denote now $\check{\imath}: S^{1} \hookrightarrow \widetilde{\mathbf{A}}^{1}$ and $\widetilde{\bar{k}}$ its complementary inclusion in $\widetilde{\mathbf{A}}^{1}$ (and similarly after taking a product with $\mathscr{X}$ or $\mathscr{X} \times \tilde{\mathbf{P}}^{1}$ ).

Let $\widetilde{L^{\prime}} \subset S^{1} \times \overline{\mathbf{A}}^{1}$ be defined by the equation $\cos (\theta+\check{\theta}) \geq 0$. We have $\widetilde{L^{\prime}}+Z \times\left[0, \infty\left[\right.\right.$ with $Z \subset S^{1} \times \breve{S}^{1}$. Let $\check{r}: \widetilde{L}^{\prime} \rightarrow L^{\prime+}$ be induced by the real blowing up of $\tau=0$. Then the natural morphism $\mathbf{Q}_{L^{\prime+}} \rightarrow \mathbb{R} \check{r}_{*} \mathbf{Q}_{\widetilde{L}^{\prime}}$ is an isomorphism: indeed, it is enough to verify this above $\tau=0$ (because $r$ is an isomorphism above $\tau \neq 0$ ); the assertion comes from the fact that the fibre of $\check{r}$ above $(\theta, 0)$ is the set of $\check{\theta}$ satisfying $\cos (\theta+\check{\theta}) \geq 0$, hence is a closed interval.

It follows from this remark that we may compute $\mathscr{F}_{X}^{\text {ir }}(\mathscr{F})$ using $\mathscr{X} \times \tilde{\mathbf{P}}^{1} \times \widetilde{\mathbf{A}}^{1}$ and $\mathscr{X} \times{\widetilde{L^{\prime}}}^{+}$with a formula analogous to (1.2). After a little manipulation, we see that it is enough to show the following, taking for $\mathscr{G}$ the inverse image of the complex $\tilde{l}^{-1} \boldsymbol{R} \tilde{k}_{*} \mathscr{F}$ by the natural map $\mathscr{X} \times Z \rightarrow \mathscr{X} \times S^{1}$ induced by the first projection:

Given any $\mathbf{R}$-constructible complex $\mathscr{G}$ on $\mathscr{X} \times Z$, consider its inverse image $\eta^{-1 \mathscr{G}}$ by the projection $\eta: \mathscr{X} \times Z \times\left[0,+\infty\left[\rightarrow \mathscr{X} \times Z\right.\right.$. Then $\widetilde{i} \eta^{-1} \mathscr{G}=0$.

By duality it is enough to verify that $\check{l}^{-1} \eta^{\prime} \mathscr{G}=0$ for any such $\mathscr{G}$. But for an open set of the form $W=V \times\left[0, \varepsilon\left[\right.\right.$ in $\mathscr{X} \times Z \times\left[0,+\infty\right.$ [, we have $\boldsymbol{R} \eta, \mathbf{Q}_{W}=0$ since the cohomology with compact support of a semi-closed interval is equal to 0 . Hence 


$$
\begin{aligned}
\boldsymbol{R} \Gamma\left(W, \eta^{!} \mathscr{G}\right) & =\boldsymbol{R} \Gamma\left(V, \boldsymbol{R} \eta_{*} \boldsymbol{R} \mathscr{H} \operatorname{om}\left(\mathbf{Q}_{W}, \eta^{!} \mathscr{G}\right)\right) \\
& =\boldsymbol{R} \Gamma\left(V, \boldsymbol{R} \mathscr{H} \circ \operatorname{om}\left(\boldsymbol{R} \eta ! \mathbf{Q}_{W}, \mathscr{G}\right)\right) \quad(\text { e.g. [11, Prop. 3.1.10]) } \\
& =0
\end{aligned}
$$

and this concludes the proof of the lemma.

Let us now sketch the proof of the second point of the theorem. Using notation of $\S 1.9$ we have

$$
{ }^{p} \psi_{\tau} \mathfrak{\mho}_{X}^{\mathrm{ir}}(\mathscr{F})=\check{i}^{-1} \boldsymbol{R} \check{k}_{*}\left(\check{k}^{-1} \mathfrak{F}_{\mathscr{X}}^{\mathrm{ir}}(\mathscr{F}) \otimes \check{\mathscr{L}}\right) .
$$

We will compute this complex using $\mathscr{X} \times \widetilde{\mathbf{A}}^{1}$ and the projection formula for the direct image $\check{\check{\pi}}: \mathscr{X} \times \check{S}^{1} \rightarrow \mathscr{X}$. First, the local system $\check{\mathscr{L}}$ extends naturally to a local system $\widetilde{\mathscr{L}}$ on $\widetilde{\overline{\mathbf{A}}^{1}}$. We then have

$$
p_{\tau} \widetilde{\mho}_{\mathscr{X}}^{\mathrm{ir}}(\mathscr{F})=\boldsymbol{R} \check{\pi}_{*}^{\check{l}} \widetilde{\boldsymbol{l}}^{-1}\left(\boldsymbol{R} \widetilde{\bar{k}}_{*}^{\widetilde{k}^{-1}} \mathfrak{\mho}_{\mathscr{X}}^{\mathrm{ir}}(\mathscr{F}) \otimes \widetilde{\breve{\mathscr{L}}}\right)
$$

Denote $\widetilde{\mathscr{F}}_{\mathscr{X}}^{\text {ir }}$ the functor defined as in (1.2) using $\widetilde{\mathbf{A}}^{1}$ instead of $\check{\mathbf{A}}^{1}$. The proof of Lemma 1.15 shows that $\widetilde{l}^{!} \tilde{F}_{\mathscr{X}}^{\mathrm{ir}}(\mathscr{F})=0$, so

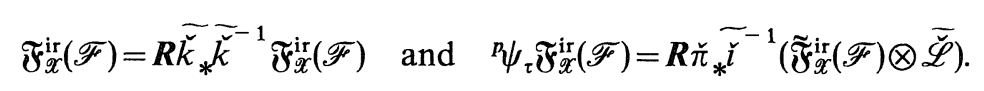

Using the projection formula for $\mathscr{X} \times \widetilde{\mathbf{P}}^{1} \times \widetilde{\mathbf{A}}^{1} \rightarrow \mathscr{X} \times \widetilde{\mathbf{A}}^{1}$ and denoting $\pi_{Z}: \mathscr{X} \times Z \rightarrow \mathscr{X}$ the projection, we get

$$
{ }^{p} \psi_{\tau} \mathfrak{F}_{\mathscr{X}}^{\mathrm{ir}}(\mathscr{F})=\boldsymbol{R} \pi_{Z *}\left(p^{-1} \boldsymbol{R} \tilde{k}_{*} \mathscr{F} \otimes \tilde{\mathscr{L}} \otimes \mathbf{Q}_{X \times Z}\right) .
$$

On the other hand, with analogous notation, we have for an object $\mathscr{F}$ of $D_{c}^{b}\left(\mathscr{X} \times \mathbf{A}^{1}\right)$ :

$$
\begin{aligned}
& { }^{p} \psi_{1 / t} \mathscr{F}=i^{-1} \boldsymbol{R} k_{*}(\mathscr{F} \otimes \mathscr{L}) \\
& =\boldsymbol{R} \tilde{\pi}_{*}^{\tilde{l}^{-1}} \boldsymbol{R} \tilde{k}_{*}\left(\mathscr{F} \otimes \tilde{k}^{-1} \tilde{\mathscr{L}}\right) \\
& =\boldsymbol{R} \tilde{\pi}_{*^{I}} \tilde{l}^{-1}\left[\boldsymbol{R} \tilde{\boldsymbol{k}}_{*} \mathscr{F} \otimes \tilde{\mathscr{L}}\right]
\end{aligned}
$$

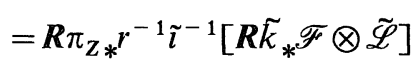

$$
\begin{aligned}
& =\boldsymbol{R} \pi_{Z *}\left[p^{-1} \boldsymbol{R} \tilde{\boldsymbol{k}}_{*} \mathscr{F} \otimes \tilde{\mathscr{L}} \otimes \mathbf{Q}_{X \times Z}\right]
\end{aligned}
$$

where $r: \mathscr{X} \times Z \rightarrow \mathscr{X} \times S^{1}$ denotes the projection, and using the isomorphism Id $\stackrel{\sim}{\rightarrow} \boldsymbol{R} r_{*} r^{-1}$, proved as for $\check{r}$. 
The assertion follows now from the identification on $Z$ of the two local systems $\widetilde{\mathscr{L}}$ and $\tilde{\mathscr{L}}$ : denote $\gamma($ resp. $\gamma)$ the generator of $S^{1}$ corresponding to $T$ (resp. the one of $\breve{S}^{1}$ corresponding to $\breve{T}$ ); the fundamental group of $Z$ is the free group generated by $\delta$ and the inclusion $Z \hookrightarrow S^{1} \times \check{S}^{1}$ maps $\delta$ to $\gamma \tilde{\gamma}$.

The compatibility of the canonical morphisms claimed in $1.10-(2)$ is a direct consequence of the previous proof and the description of these morphisms given in $\$ 1.9$.

\section{§2. Fourier Transform of Regular Holonomic $\mathscr{D}$-modules and a Comparison Theorem}

In this section $\mathscr{X}$ denotes a smooth quasi-projective variety equipped with its Zariski topology, or an analytic manifold. The lines $\mathbb{A}^{1}$ and $\check{\mathbb{A}}^{1}$ will be equipped with their Zariski topology, so for instance $\mathscr{D}_{\mathscr{X} \times \mathbf{A}^{1}}$-modules will be $\mathscr{D}_{\mathscr{X}}[t]\left\langle\partial_{t}\right\rangle$-modules.

We consider the Zariski topology when dealing with $\mathcal{O}$ or $\mathscr{D}$-modules and the analytic topology when dealing with constructible sheaves.

2.1. Fourier transform of $\mathscr{D}$-modules on the affine line. Recall the notion of Fourier transform (or better Laplace transform, the kernel being $e^{-t \tau}$ ) of a holonomic $\mathbb{C}[t]\left\langle\partial_{t}\right\rangle$-module (see [14] for details). Let $M$ be a left $\mathbb{C}[t]\left\langle\partial_{t}\right\rangle$-module of finite type. Identify the two rings $\mathbb{C}[t]\left\langle\partial_{t}\right\rangle$ and $\mathbb{C}[\tau]\left\langle\partial_{\tau}\right\rangle$ via the isomorphism $t \mapsto-\partial_{\tau}, \partial_{t} \mapsto \tau$. Denote $\widehat{M}$ the module $M$ when viewed as a left $\mathbb{C}[\tau]\left\langle\partial_{\tau}\right\rangle$-module via this isomorphism. Then $\widehat{M}$ is the Fourier transform of $M$.

Assume that $M$ has only regular singularities, even at infinity. Then $\widehat{M}$ has singularities at $\tau=0$ and $\tau=\infty$ only, the singularity at $\tau=0$ being regular. The singularity at $\tau=\infty$ is not regular if $M$ has singularities at $t \neq 0$, $\infty$. Recall the notation of projections

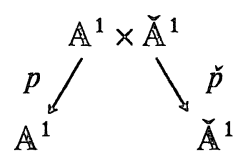

Let $M[\tau]=M \otimes_{\mathbb{C}} \mathbb{C}[\tau]=p^{+} M$ be the inverse image of $M$ on $\mathbb{A}^{1} \times \check{\mathbb{A}}^{1}$ with its natural structure of $\mathbb{C}[t, \tau]\left\langle\partial_{t}, \partial_{\tau}\right\rangle$-module and $M[\tau] \otimes e^{-t \tau}$ be the same $\mathbb{C}[t, \tau]$-module with the following twisted action of $\partial_{t}$ and $\partial_{\tau}$, for $m \in M[\tau]$ : 


$$
\partial_{t}\left(m \otimes e^{-t \tau}\right)=\left[\left(\partial_{t}-\tau\right) m\right] \otimes e^{-t \tau}, \quad \partial_{\tau}\left(m \otimes e^{-t \tau}\right)=\left[\left(\partial_{\tau}-t\right) m\right] \otimes e^{-t \tau}
$$

The direct image $\check{p}_{+}\left(M[\tau] \otimes e^{-t \tau}\right)$ is the complex

$$
M[\tau] \stackrel{\partial_{t}-\tau}{\longrightarrow} M[\tau]
$$

where the right hand term has degree 0 , and this complex has cohomology in degree 0 only, the cokernel of $\partial_{t}-\tau$ being identified with $\hat{M}$ by the map

$$
\begin{gathered}
M[\tau] \rightarrow M \\
\sum_{i} m_{i} \tau^{i} \mapsto \sum_{i} \partial_{t}^{i} m_{i} .
\end{gathered}
$$

We shall write $\hat{M}=\check{p}_{+}\left(M[\tau] \otimes e^{-t \tau}\right)=\check{p}_{+}\left(p^{+} M \otimes e^{-t \tau}\right)$.

Analogous results hold for a bounded complex $M^{\bullet}$ with regular holonomic cohomology (regularity is used here only to analyse the singularities at finite distance of $\hat{M}^{\circ}$ ).

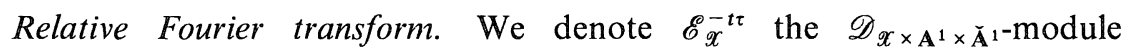

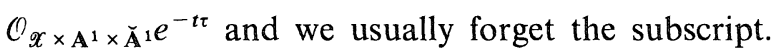

Let $\mathscr{M}$ be a holonomic $\mathscr{D}_{\mathscr{X} \times \mathbf{A}^{1} \text {-module (or a bounded complex with such }}$ a cohomology). Its Fourier transform is the object

$$
\widetilde{\mho}_{\mathscr{X}}(\mathscr{M}) \stackrel{\text { def }}{=} \check{p}_{+}\left(p^{+} \mathscr{M} \otimes \mathscr{E}^{-t \tau}\right)
$$

of $D_{\text {hol }}^{b}\left(\mathscr{D}_{\mathscr{X} \times \check{\mathbf{A}}^{1}}\right)$. When $\mathscr{M}$ is a single holonomic $\mathscr{D}_{\mathscr{X}}[t]\left\langle\partial_{t}\right\rangle$-module, $\mathfrak{F}_{\mathscr{X}}(\mathscr{M})$ is the single holonomic $\mathscr{D}_{\mathscr{X}}[\tau]\left\langle\partial_{\tau}\right\rangle$-module obtained from $\mathscr{M}$ as in $\S 2.1$.

Let $F: \mathscr{X} \rightarrow \mathbf{P}^{1}$ be a rational function and still denote $F$ its restriction $F_{\mid X}: X \rightarrow \mathbf{A}^{1}$. For $\mathscr{M}$ holonomic on $X$, define $\widetilde{\mho}_{F}(\mathscr{M})=k_{F+}\left(p^{+} \mathscr{M} \otimes \mathscr{E}^{-\tau F}\right)$, with $\mathscr{E}^{-\tau F}=\left(F_{\mid X} \times \mathrm{Id}\right)^{+} \mathscr{E}^{-\tau t}=\mathcal{O}_{X \times \check{\mathbf{A}}^{1}} e^{-\tau F}$. We have $\mathfrak{F}_{F}(\mathscr{M})=\mathfrak{F}_{X}\left(i_{F+}^{\prime} \mathscr{M}\right)$.

For $j: U \hookrightarrow X$ the inclusion of a Zariski open set, we denote $f=F_{\mid X} \circ j=F_{\mid U}$, and we have $\widetilde{\mho}_{F}\left(j_{+} \mathfrak{O}_{U}\right)=\kappa_{+} \mathscr{E}^{-\tau f}$ with $\kappa=k_{F} \circ j$ and $\mathscr{E}^{-\tau f}=\mathfrak{O}_{U \times \mathbf{A}^{1}} e^{-\tau f}$.

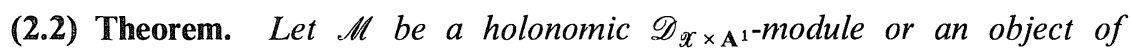
$D_{\text {hol }}^{b}\left(\mathscr{D}_{\mathscr{X} \times \mathbf{A}^{1}}\right)$ such that $k_{+} \mathscr{M}$ has $\mathscr{D}_{\mathscr{X} \times \mathbb{P}^{1}}$-regular holonomic cohomology. We have a functorial isomorphism

$$
{ }^{p} \mathrm{DR}^{\mathrm{an}} \mathfrak{F}_{\mathscr{X}}(\mathscr{M}) \simeq \mathfrak{F}_{X^{p}} \mathrm{DR}^{\mathrm{an}}(\mathscr{M}) \quad \text { in } D_{c}^{b}\left(\mathbf{C}_{\left.\mathscr{X} \times \check{\mathrm{A}}^{1}\right)}\right.
$$




\subsection{Remarks.}

1. It is possible to extend this theorem to the case where $\mathscr{X}$ is analytic. One needs to define the Fourier transform as a functor from

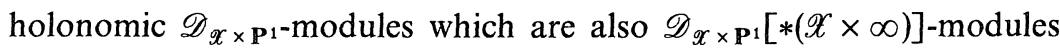

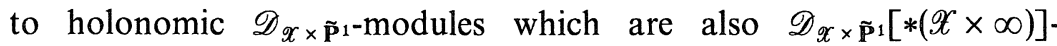
modules. These tools have been developed in [1].

2. This theorem is proved (but not stated explicitely, and for the solution complex instead of the de Rham complex) in [5, $\S 3.3,3.4,3.5]$ under a weaker assumption: $k_{+} \mathscr{M}$ should be "1-specializable" along $t=\infty$. This is an assumption of regularity along $t=\infty$. We will give below another proof by reduction to dimension 1 in the regular holonomic case, analogous to the one of [22, th. 5.1].

3. When considering $f: U \rightarrow \mathbf{A}^{1}$ and the $\mathscr{D}_{U}$-module $\mathscr{O}_{U}$, this comparison theorem gives a rational structure on ${ }^{p} \mathrm{DR}^{\mathrm{an}}\left(\kappa_{+} \mathscr{E}^{-\tau f}\right)$ by the isomorphism

$$
{ }^{p} \mathrm{DR}^{\mathrm{an}} \mathfrak{F}_{F}\left(j_{+} \mathcal{O}_{U}\right) \simeq \mathfrak{F}_{F}\left(\boldsymbol{R} j_{*}^{p} \mathbf{Q}_{U}\right) \underset{\mathbf{Q}}{\otimes \mathbf{C}}
$$

4. The Fourier functor commutes with direct image for morphisms $\mathscr{X} \rightarrow \mathscr{Y}$ of smooth projective varieties. In particular, the Fourier transform of the Gauss-Manin system $f_{+} \mathcal{O}_{U}$ is equal to $\check{p}_{+} \kappa_{+} \mathscr{E}^{-\tau f}$, if $\check{p}$ denotes here the projection $\mathscr{X} \times \check{\mathbf{A}}^{1} \rightarrow \check{\mathbf{A}}^{1}$.

5. The weight fltration. Assume now that $X-U$ is a divisor with normal crossings in $X$. Then the perverse complex $\boldsymbol{R} j_{*}{ }^{p} \mathbf{Q}_{U}$ is filtered by perverse subobjects $W \cdot \boldsymbol{R}_{*}{ }^{p} \mathrm{Q}_{U}$ in the category of perverse sheaves on $X([6]$ and [2]). This filtration corresponds to the filtration by the number of polar components on the regular holonomic $\mathscr{D}_{X}$-module $j_{+} \mathcal{O}_{U}=\mathcal{O}_{X}[*(X-U)]$, via the functor ${ }^{p} \mathrm{DR}^{\text {an }}$. The Fourier transform of $\mathscr{H}^{i} F_{+}\left(W_{\bullet} j_{+} \mathcal{O}_{U}\right)$ is equal to $\mathscr{H}^{i} \check{p}_{+}\left(W_{\bullet} j_{+} \mathcal{O}_{U}\right.$ $\otimes \mathscr{E}^{-\tau F}$ ), and, by functoriality, the Fourier transform of the filtration

$$
W \cdot \mathscr{H}^{i} f_{+} \mathcal{O}_{U} \stackrel{\text { def }}{=} \text { image } \mathscr{H}^{i} F_{+}\left(W_{\bullet} j_{+} \mathcal{O}_{U}\right) \rightarrow \mathscr{H}^{i} f_{+} \mathcal{O}_{U}
$$

is equal to the filtration

$$
W_{\bullet} \mathscr{H}^{i} \widehat{f_{+} \mathcal{O}_{U}} \stackrel{\text { def }}{=} \text { image } \mathscr{H}^{i} \check{p}_{+}\left(W_{\bullet} j_{+} \mathcal{O}_{U} \otimes \mathscr{E}^{-\tau F}\right) \rightarrow \mathscr{H}^{i} \check{p}_{+} \mathscr{E}^{-\tau f}
$$

Notice also that when $U$ is quasi-projective, such a filtration does not 
depend on the choice of a quasi-projective compactification $X \rightarrow \mathbf{A}^{1}$ of $f$ because this is the weight filtration of the mixed Hodge module associated with $f_{+} \mathfrak{O}_{U}$ [24].

\section{Proof of Theorem 2.2 .}

(1) As ${ }^{p} \mathrm{DR}^{\mathrm{an}}$ commutes with direct image by $\check{p}$ (see e.g. [19, II.5.5]), it is enough to find a functorial isomorphism

$$
{ }^{p} \mathrm{DR}^{\mathrm{an}} k_{+}\left(p^{+} \mathscr{M} \otimes \mathscr{E}^{-\tau t}\right) \stackrel{\sim}{\rightarrow} \boldsymbol{R} \varepsilon_{*}\left(\boldsymbol{R} \tilde{k}_{*} p^{-1 p} \mathrm{DR}^{\mathrm{an}}(\mathscr{M})[1] \otimes \mathbf{Q}_{\mathscr{X} \times L^{-}}\right)
$$

We first lift the LHS as a complex ${ }^{p} \mathrm{DR}^{\bmod } k_{+}\left(p^{+} \mathscr{M} \otimes \mathscr{E}^{-\tau t}\right)$ on $\mathscr{X} \times \widetilde{\mathbf{P}}^{1} \times \check{\mathbf{A}}^{1}$ such that

$$
\boldsymbol{R} \varepsilon_{*}^{p} \mathrm{DR}^{\mathrm{mod}} k_{+}\left(p^{+} \mathscr{M} \otimes \mathscr{E}^{-\tau t}\right)={ }^{p} \mathrm{DR}^{\mathrm{an}} k_{+}\left(p^{+} \mathscr{M} \otimes \mathscr{E}^{-\tau t}\right) .
$$

It will then be enough to find a morphism

$$
{ }^{p} \mathrm{DR}^{\bmod } k_{+}\left(p^{+} \mathscr{M} \otimes \mathscr{E}^{-\tau t}\right) \rightarrow R \tilde{k}_{*} p^{-1 p} \mathrm{DR}^{\text {an }}(\mathscr{M})[1] \otimes Q_{\mathscr{C} \times L^{-}}
$$

and to prove that it is an isomorphism.

Let $\mathscr{A}_{\mathbf{P}^{1} \times \check{\mathbf{A}}^{1}}^{\text {mod }}$ be the sheaf on $\widetilde{\mathbf{P}}^{1} \times \breve{\mathbf{A}}^{1}$ of functions which are holomorphic on $\mathbf{A}^{1} \times \check{\mathbf{A}}^{1}$ and have moderate growth along $S^{1} \times \check{\mathbf{A}}^{1}$, where $S^{1}=\varepsilon^{-1}(\infty)$. This is a flat module over $\varepsilon^{-1} \mathcal{O}_{\mathbf{P}^{1} \times \check{\mathbf{A}}^{1}}^{\text {an }}$ (the proof is identical to the one of Prop. 2.8 in [21]). This sheaf comes also equipped with a natural $\varepsilon^{-1} \mathscr{D}_{\mathbf{P}^{1} \times \mathbf{A}^{1}}^{\text {an }}$-module structure. Let $F$ be the projection of $\mathscr{X} \times \mathbf{P}^{1} \times \check{\mathbf{A}}^{1}$ on $\mathbf{P}^{1} \times \check{\mathbf{A}}^{1}$. Then $F^{-1} \mathscr{A}_{\mathbf{P}^{1} \times \check{\mathbf{A}}^{1}}^{\text {mod }} \otimes_{\varepsilon^{-1} F^{-1} \mathscr{O}_{\mathbf{P}^{1} \times \check{\mathbf{A}}_{1}}} \varepsilon^{-1} \mathscr{M}$ has a natural structure of a left $\varepsilon^{-1} \mathscr{D}_{\mathscr{X} \times \mathbf{P}^{1} \times \check{\mathbf{A}}^{1^{-}}}^{\text {an }}$ module if $\mathscr{M}$ is a left $\mathscr{D}_{\mathscr{C}} \times \mathbf{P}^{1} \times \check{\mathbf{A}}^{1}$-module. Moreover we have

$$
\boldsymbol{R} \varepsilon_{*} \mathscr{A} \underset{\mathbf{P} 1 \times \mathbf{A}^{1}}{\bmod }=\mathscr{O}_{\mathbf{P}^{1} \times \check{\mathbf{A}}^{1}}^{\text {an }}\left[*\left(\{\infty\} \times \check{\mathbf{A}}^{1}\right)\right]
$$

(see [14, Lemma 3.4] in dimension 1 and the proof of Th. 5.1 in [22] for the case considered above).

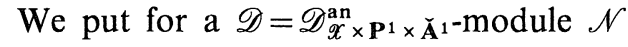

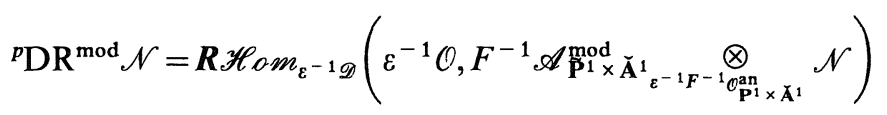

which satisfies $R \varepsilon_{*}^{p} \mathrm{DR}^{\text {mod }} \mathscr{N}={ }^{p} \mathrm{DR}^{\text {an }}\left(k_{+} k^{+} \mathcal{N}\right)$ by the projection formula and (2.6).

(2) Let $\gamma: L^{\prime+} \varsigma \widetilde{\mathbf{P}}^{1} \times \breve{\mathbf{A}}^{1}(\times \mathscr{X})$ be the closed inclusion complementary to 
$\beta$ (see $\S 1.5$ ). To obtain the morphism (2.5) it is enough to prove that

$$
\gamma^{-1 p} \mathrm{DR}^{\mathrm{mod}} k_{+}\left(p^{+} \mathscr{M} \otimes \mathscr{E}^{-\tau t}\right)=0 .
$$

This is a local problem on $\mathscr{X}^{\text {an }}$. If this is proved, one has, putting $\mathscr{K}={ }^{p} \mathrm{DR}^{\mathrm{mod}} k_{+}\left(p^{+} \mathscr{M} \otimes \mathscr{E}^{-\tau t}\right)$,

$$
\mathscr{K} \leftarrow \beta_{!} \beta^{-1} \mathscr{K} \rightarrow \beta_{!} \mathbb{R} \alpha_{*} \alpha^{-1} \beta^{-1} \mathscr{K}=\beta_{!} \mathbb{R} \alpha_{*} k^{-1} \mathscr{K} \simeq \beta_{!} \mathbb{R} \alpha_{*} p^{-1 p} \operatorname{DR}^{a n}(\mathscr{M})
$$

and this last term is exactly the RHS in (2.5). Now, the fact that this morphism is an isomorphism is also a local problem on $\mathscr{X}^{\text {an }}$.

(3) When $\mathscr{X}=\mathrm{pt},(2.7)$ and the fact that (2.5) is an isomorphism is essentially proved in $[14, \S \mathrm{V} .3]$. As $k_{+} \mathscr{M}$ is regular and as the problem is local on $S^{1} \times \check{\mathrm{A}}^{1}$, one first reduces to the case where $\left(k_{+} \mathscr{M}\right)^{\text {an }}$ is a rank one meromorphic connection near $\infty$, and locally on $S^{1} \times \check{\mathbb{A}}^{1}$ one may reduce to the trivial meromorphic connection $\mathcal{O}[* \infty]$ because $t^{\alpha}$ defines a local invertible section of $\mathscr{A}^{\text {mod }}$. Let $t^{\prime}=1 / t$ be the local coordinate on a disc near $\infty$. The statement is then equivalent to ${ }^{p} \mathbb{D} R^{\bmod }\left(\mathcal{O}_{D \times \check{A}^{\text {an }}}^{\text {an }} e^{-\tau / t^{\prime}}\right)=\mathbb{C}_{L^{-}}[2]$, or in other words, the single complex made with the following double complex

$$
\begin{aligned}
& \mathscr{A}_{\overline{\bar{D}} \times \overline{\mathbb{A}}^{1}}^{\bmod } e^{-\tau / t^{\prime}} \stackrel{\partial_{\mathcal{L}}}{\rightarrow} \mathscr{A}_{\overline{\bar{D}} \times \check{\mathbb{A}}^{1}}^{\bmod } e^{-\tau / t^{\prime}} \\
& \partial_{t^{\prime}} \downarrow \quad \downarrow \partial_{t^{\prime}}
\end{aligned}
$$

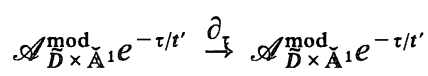

has cohomology in degree 0 only, if the upper left corner has bidegree $(0,0)$.

(4) We will now reduce the problem to the case $\mathscr{X}=\mathrm{pt}$ by taking a local direct image by the projection $F: \mathscr{X} \times \mathbb{P}^{1} \rightarrow \mathbb{P}^{1}$. Let $D$ be a small disc around $\infty \in \mathbb{P}^{1}$ and $B\left(x^{0}, \rho\right)$ the closed ball of radius $\rho>0$ (for some metric) centered at $x^{0} \in \mathscr{X}$ and denote $F_{x^{0}, \rho}: B\left(x^{0}, \rho\right) \times D \rightarrow D$ the restriction of $F$. Recall (see Th. 9.4.1 and Cor. 9.4.2 in [10]) that, given a regular holonomic module or bounded complex $\mathscr{N}^{\text {an }}$, the local direct image $F_{x^{0}, \rho+} \mathscr{N}^{\text {an }}$ has $\mathscr{D}_{D^{-}}$-regular holonomic cohomology for $\rho$ and $D$ small enough. Remark also that, if $\mathscr{N}^{\text {an }}=\mathscr{N}^{\text {an }}\left[t^{\prime-1}\right]$, each cohomology module $N$ is a meromorphic connection on $D$ with pole at $\infty$, i.e. is equal to $N\left[t^{\prime-1}\right]$. It follows that for $\mathscr{M}$ as 
above, the cohomology modules of

$$
F_{x^{0}, \rho+}\left(p^{+} k_{+} \mathscr{M} \otimes \mathscr{E}^{-\tau t}\right)^{\mathrm{an}}=\left(p^{+} F_{x^{0}, \rho+}\left(k_{+} \mathscr{M}\right)^{\mathrm{an}}\right) \otimes \mathscr{E}^{-\tau / t^{\prime}}
$$

(where $p^{+}$is taken in the analytic sense in the RHS) are meromorphic connections on $D \times \check{\mathbf{A}}^{1}$ with poles along $t^{\prime} \tau=0$. Notice that step (3) gives the isomorphism (2.5) for $F_{x^{0}, \rho+}\left(k_{+} \mathscr{M}\right)^{\text {an }}$.

As $F_{x^{0}, \rho}$ is proper and according to the description of (2.5) given in step (2), it is then enough to find an isomorphism

$$
\boldsymbol{R} F_{x^{0}, \rho *}^{p} \mathrm{DR}^{\mathrm{mod}}\left(p^{+} k_{+} \mathscr{M} \otimes \mathscr{E}^{-\tau t}\right) \stackrel{\sim}{\rightarrow}{ }^{p} \mathrm{DR}^{\mathrm{mod}} F_{x^{0}, \rho+}\left(p^{+} k_{+} \mathscr{M} \otimes \mathscr{E}^{-\tau t}\right)^{\mathrm{an}}
$$

This is done as for the analytic de Rham complex (see e.g. [19, §II.5.5]).

\section{§3. Regularity of $\mathfrak{F}_{F}\left(j_{+} \mathcal{O}_{U}\right)$ along $\tau=0$}

We keep notation of Section 2. We shall show in this section that the

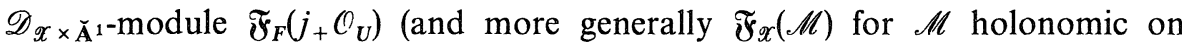
$\mathscr{X} \times \mathbf{A}^{1}$ and $k_{+} \mathscr{M}$ regular on $\left.\mathscr{X} \times \mathbf{P}^{1}\right)$ is strongly regular along $\tau=0$. To this end, we first recall the main properties of the Malgrange-Kashiwara $V$-filtration of a holonomic $\mathscr{D}$-module along $\tau=0$ as well as a criterion of regularity along $\tau=0$, which allows one to express the nearby and vanishing cycles ${ }^{p} \psi_{\tau}{ }^{p} \mathrm{DR}^{\text {an }} \mathfrak{F}_{F}\left(j_{+} \mathcal{O}_{U}\right)$ and ${ }^{p} \phi_{\tau}{ }^{p} \mathrm{DR}^{\mathrm{an}} \mathfrak{F}_{F}\left(j_{+} \mathcal{O}_{U}\right)$ as de Rham complexes of holonomic $\mathscr{D}_{\mathscr{X}}$-modules computed in terms of the $V$-filtration.

Remark however that $\mathfrak{F}_{F}\left(j_{+} \mathcal{O}_{U}\right)=\kappa_{+} \mathscr{E}^{-\tau f}$ is not a regular $\mathscr{D}$-module, but its irregularity is concentrated along $F^{-1}(\infty) \times \check{\AA}^{1}$ in a neighbourhood of $\tau=0$. The nontrivial part of the monodromy of ${ }^{p} \psi_{\tau}{ }^{p} \mathrm{DR}^{\mathrm{an}} \widetilde{\mho}_{F}\left(j_{+} \mathcal{O}_{U}\right)$ comes from the monodromy around $\tau=0$ of the irregularity sheaf of $\mathfrak{F}_{F}\left(j_{+} \mathcal{O}_{U}\right)$.

3.1. The Malgrange-Kashiwara filtration of a holonomic $\mathscr{D}$-module. Let $\mathscr{X}$ be a smooth algebraic or analytic variety and let $\mathscr{D}_{\mathscr{X}}$ be the corresponding sheaf of differential operators on $\mathscr{X}$. Let $V_{\bullet} \mathscr{D}_{\mathscr{X}}[\tau]\left\langle\partial_{\tau}\right\rangle$ be the increasing filtration indexed by $\mathbb{Z}$ such that

$$
\begin{aligned}
& V_{0} \mathscr{D}_{\mathscr{X}}[\tau]\left\langle\partial_{\tau}\right\rangle=\mathscr{D}_{\mathscr{X}}[\tau]\left\langle\tau \partial_{\tau}\right\rangle, \\
& V_{k} \mathscr{D}_{\mathscr{X}}[\tau]\left\langle\partial_{\tau}\right\rangle=V_{k-1}+\partial_{\tau} V_{k-1} \quad \text { for } k \geq 1, \\
& V_{k} \mathscr{D}_{\mathscr{X}}[\tau]\left\langle\partial_{\tau}\right\rangle=\tau^{-k} V_{0} \mathscr{D}_{\mathscr{X}}[\tau]\left\langle\partial_{\tau}\right\rangle \quad \text { for } k \leq 0 .
\end{aligned}
$$


Let $\mathscr{M}$ be a holonomic $\mathscr{D}_{\mathscr{X}}[\tau]\left\langle\partial_{\tau}\right\rangle$-module. There exists a unique increasing filtration $V_{\mathscr{A}} \mathscr{M}$, called the Malgrange-Kashiwara filtration of $\mathscr{M}$, which is indexed by a finite number of lattices $\alpha+\mathbf{Z} \subset \mathbb{C}$ (in general one has to fix a total order on $\mathbb{C}$, but, for our purpose, it will be enough to assume that the lattices are contained in $\mathbf{Q}$, i.e. that the roots of the corresponding Bernstein polynomial are rational) which satisfies the following properties:

1. For each $\alpha$, the filtration $V_{a+k} \mathscr{M},(k \in \mathbb{Z})$, is good with respect to $V . \mathscr{D}_{\mathscr{X}}[\tau]\left\langle\partial_{\tau}\right\rangle$;

2. for every $\beta$, there exists locally on $\mathscr{X}$ an integer $\delta_{\beta}$ such that $\left(\tau \partial_{\tau}+\beta\right)^{\delta_{\beta}} V_{\beta} \mathscr{M} \subset V_{<\beta} \mathscr{M}$, where $V_{<\beta}=\underset{\beta^{\prime}<\beta}{\cup} V_{\beta^{\prime}}$.

We shall denote $\operatorname{gr}_{\beta}^{V} \mathscr{M}$ the quotient $V_{\beta} \mathscr{M} / V_{<\beta} \mathscr{M}$. This is a holonomic $\mathscr{D}_{\mathscr{X}}$-module equipped with a nilpotent endomorphism $N$ induced by the action of $\tau \partial_{\tau}+\beta$.

Remark. In [20] or [23] the filtration denoted $V$ is the previous filtration shifted by 1 . The choice made here will be convenient for our purpose.

The moderate nearby or vanishing cycle functor $\psi_{\tau}^{\bmod }$ or $\phi_{\tau}^{\bmod }$ for holonomic $\mathscr{D}$-modules is defined by

$$
\begin{aligned}
& \left.\psi_{\tau, \lambda}^{\mathrm{mod}} \mathscr{M}=\phi_{\tau, \lambda}^{\mathrm{mod}} \mathscr{M}=\operatorname{gr}_{\alpha}^{V} \mathscr{M} \text { with } \alpha \in\right] 0,1[\text { and } \lambda=\exp (2 i \pi \alpha) \neq 1 \\
& \psi_{\tau, 1}^{\mathrm{mod}} \mathscr{M}=\operatorname{gr}_{0}^{V} \mathscr{M}, \quad \phi_{\tau, 1}^{\mathrm{mod}} \mathscr{M}=\operatorname{gr}_{1}^{V} \mathscr{M} \\
& \psi_{\tau}^{\mathrm{mod}} \mathscr{M}=\underset{\lambda \in \mathbf{C}^{*}}{\bigoplus} \psi_{\tau, \lambda}^{\bmod } \mathscr{M}, \quad \phi_{\tau}^{\bmod } \mathscr{M}=\underset{\lambda \in \mathbf{C}^{*}}{\bigoplus} \phi_{\tau, \lambda}^{\bmod } \mathscr{M}
\end{aligned}
$$

which is a functor from holonomic $\mathscr{D}_{\mathscr{X}}[\tau]\left\langle\partial_{\tau}\right\rangle$-modules to $\mathscr{D}_{\mathscr{X}}\left[T, T^{-1}\right]$-modules which are $\mathscr{D}_{\mathscr{X}}$-holonomic, where $T=\lambda \exp (-2 i \pi N): \psi_{\tau, \lambda}^{\bmod } \mathscr{M} \rightarrow \psi_{\tau, \lambda}^{\bmod } \mathscr{M}$ or $\phi_{\tau, \lambda}^{\bmod } \mathscr{M} \rightarrow \phi_{\tau, \lambda}^{\bmod } \mathscr{M}$. There are morphisms

$$
\operatorname{can}: \psi_{\tau, 1}^{\bmod } \mathscr{M} \rightarrow \phi_{\tau, 1}^{\bmod } \mathscr{M} \quad \text { and } \quad \operatorname{var}: \phi_{\tau, 1}^{\bmod } \mathscr{M} \rightarrow \psi_{\tau, 1}^{\bmod } \mathscr{M}
$$

defined by can $=-\partial_{\tau} \sum_{n \geq 1} \frac{(2 i \pi)^{n}}{n !} \cdot\left(-\tau \partial_{\tau}\right)^{n-1}$ and var $=\tau$, such that can $\circ$ var $=T-$ Id and var $\circ$ can $=T$-Id. For $\lambda \neq 1$ we also define can $=T-$ Id $: \psi_{\tau, \lambda}^{\bmod } \mathscr{M} \rightarrow \psi_{\tau, \lambda}^{\bmod } \mathscr{M}$ and $\operatorname{var}=\mathrm{Id}$. 
Assume that $\mathscr{X}$ is algebraic and let $\mathscr{X}^{\text {an }}$ be the underlying analytic manifold. For a $\mathscr{D}_{\mathscr{X}}$-module $\mathscr{M}$, let $\mathscr{M}^{\text {an }}=\mathcal{O}_{\mathscr{X}}^{\text {an }} \otimes_{\mathcal{O}_{\mathscr{X}}} \mathscr{M}$. The two possible $V$-filtrations on $\mathscr{M}^{\text {an }}$ coincide:

(3.2) Lemma. For $\mathscr{M}$ holonomic over $\mathscr{D}_{\mathscr{X}}[\tau]\left\langle\partial_{\tau}\right\rangle$, we have

$$
\mathcal{O}_{\mathscr{X} \times \mathbf{A}^{1}}^{\text {an }} \underset{\mathcal{O}_{\mathscr{C} \times \overline{\mathbf{A}}^{1}}}{\otimes} V \cdot \mathscr{M}=V \cdot \mathscr{M}^{\text {an }}
$$

Proof. By flatness of $\mathcal{O}_{\mathscr{X} \times \check{\mathbf{A}}^{1}}^{\text {an }}$ over $\mathcal{O}_{\mathscr{X} \times \check{\mathbf{A}}^{1}}$ one verifies that the LHS is a good filtration of $\mathscr{M}^{\text {an }}$ (with respect to $V_{\bullet} \mathscr{D}_{\mathscr{X} \times \check{A}^{1}}^{\text {an }}$ ), satisfying the same properties as the RHS, so we get the lemma using the uniqueness of such filtration.

In particular, we have for all $\beta$,

$$
\left(\operatorname{gr}_{\beta}^{V} \mathscr{M}\right)^{\text {an }}=\operatorname{gr}_{\beta}^{V} \mathscr{M}^{\text {an }}
$$

Regularity along a smooth hypersurface. Let us keep notation as above. Let $\check{k}: \mathscr{X} \times \check{\mathbf{A}}^{1^{*}} \varsigma \mathscr{X} \times \check{\mathbf{A}}^{1}$ and $\check{\imath}: \mathscr{X} \times\{0\} \hookrightarrow \mathscr{X} \times \check{\mathbf{A}}^{1}$ denote the complementary inclusions. Following Mebkhout [18], we say that $\mathscr{M}$ is regular along $\mathscr{X} \times\{0\}$ if one of the following two equivalent conditions is satisfied:

1. the natural morphism ${ }^{p} \mathrm{DR}^{\mathrm{an}}\left(\check{k}_{+} \check{k}^{+} \mathscr{M}\right) \rightarrow \boldsymbol{R} \breve{k}_{*} \check{k}^{-1}{ }^{p} \mathrm{DR}^{\mathrm{an}}(\mathscr{M})$ is a quasiisomorphism;

2. the natural morphism $\check{l}^{-1 p} \mathrm{DR}^{\text {an }}(\mathscr{M})[1] \rightarrow{ }^{p} \mathrm{DR}^{\text {an }}\left(\check{l}^{\dagger} \mathscr{M}\right)$ is a quasiisomorphism.

Remark. The first definition can be used to define regularity along a nonnecessarily smooth hypersurface.

For $\lambda \in \mathbf{C}^{*}$, let $\mathscr{N}_{\lambda}$ be the rank one meromorphic connection on $\mathscr{X} \times \check{\mathbf{A}}^{1}$ with poles along $\mathscr{X} \times\{0\}$ generated by $\tau^{\alpha}$, where $e^{2 i \pi \alpha}=\lambda$ (such a connection depends only on $\lambda$ and not on the choice of $\alpha$ ).

We shall say that $\mathscr{M}$ is strongly regular along $\mathscr{X} \times\{0\}$ if $\mathscr{M} \otimes \mathscr{N}_{\lambda}$ is regular along $\mathscr{X} \times\{0\}$ for any $\lambda \in \mathbf{C}^{*}$.

\section{Remarks.}

1. It is not known if both conditions (regularity and strong regularity 
along $\mathscr{X} \times\{0\})$ are equivalent, but this is a reasonable conjecture.

2. Regularity (or strong regularity) of $\mathscr{M}$ along $\mathscr{X} \times\{0\}$ is equivalent to regularity (or strong regularity) of the localized module $\mathscr{M}\left[\tau^{-1}\right]$.

One can define in an analogous way the notion of regularity or strong regularity for a bounded complex with holonomic cohomology. Thanks to the theorem of Mebkhout [18], saying that the irregularity complex along $\mathscr{X} \times\{0\}$ of a holonomic $\mathscr{D}$-module is a perverse complex, the regularity (or strong regularity) of such a bounded complex is equivalent to the regularity (or strong regularity) of each of its cohomology modules (for the strong regularity statement, one uses the fact that $\mathcal{N}_{\lambda}$ is $\mathcal{O}$-flat).

Regularity or strong regularity is stable under direct image by

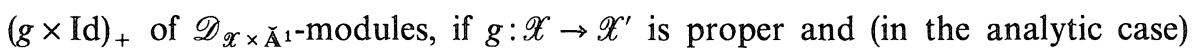
if $\mathscr{M}$ is generated by a coherent $\mathcal{C}_{x}[\tau]$-module. We have (see e.g. [20], [17, $\S 4],[13])$

(3.3) Theorem (Comparison theorem for nearby and vanishing cycles)。 Let $\mathscr{M}$ be a holonomic $\mathscr{D}_{\mathscr{X}}[\tau]\left\langle\partial_{\tau}\right\rangle$-module. Assume that $\mathscr{M}$ is strongly regular along $\tau=0$. Then there are functorial isomorphisms in $D_{c}^{b}\left(\mathbb{C}\left[T, T^{-1}\right]_{\mathscr{X}}\right)$

$$
{ }^{p} \mathrm{DR}^{\mathrm{an}}\left(\psi_{\tau}^{\mathrm{mod}} \mathscr{M}\right) \stackrel{\sim}{\rightarrow}{ }^{p} \psi_{\tau}^{p} \mathrm{DR}^{\mathrm{an}}(\mathscr{M}) \quad \text { and } \quad{ }^{p} \mathrm{DR}^{\mathrm{an}}\left(\phi_{\tau, 1}^{\mathrm{mod}} \mathscr{M}\right) \stackrel{\sim}{\rightarrow}{ }^{p} \phi_{\tau, 1}{ }^{p} \mathrm{DR}^{\mathrm{an}}(\mathscr{M})
$$

compatible with the morphisms can and var.

(3.4) Proposition. Let $\mathscr{M}$ be a holonomic $\mathscr{D}_{\mathscr{X} \times \mathbb{A}^{1}-\text { module }}$ such that $k_{+} \mathscr{M}$ is a regular $\mathscr{D}_{\mathscr{X} \times \mathbb{P}^{1}-\text { module. }} \quad$ Then $\mathfrak{F}_{X}(\mathscr{M})$ is strongly regular along $\tau=0$.

(3.5) Corollary. The complex ${ }^{p} \mathrm{DR}^{\mathrm{an}} \phi_{\tau}^{\bmod } \mathfrak{F}_{F}\left(j_{+} \mathcal{O}_{U}\right)$ admits a natural Q-structure:

$$
{ }^{p} \mathrm{DR}^{\mathrm{an}} \phi_{\tau}^{\mathrm{mod}} \widetilde{\mho}_{F}\left(j_{+} \mathcal{O}_{U}\right) \simeq{ }^{p} \phi_{\tau}\left[\widetilde{\mho}_{F}\left(\boldsymbol{R} j_{*}{ }^{p} \mathrm{Q}_{U}\right)\right] \otimes \mathbb{C}
$$

(and the same result holds for $\psi_{\tau, 1}^{\text {mod }}$ and ${ }^{p} \psi_{\tau, 1}$ ). Moreover, for different compactifications, the $\mathbb{Q}$-structures are compatible in a natural way.

Proof of Proposition 3.4. It is equivalent to prove the result for $\mathfrak{F}_{F}(\mathscr{M})$, if $F: \mathscr{X} \rightarrow \mathbb{P}^{1}$ is a meromorphic function and $\mathscr{M}$ a holonomic $\mathscr{D}_{X}$-module. Using 
resolution of singularities and a standard "dévissage", we may assume that $\mathscr{M}=\kappa_{+} \mathcal{N}$, where $\mathscr{N}$ is $\mathcal{O}$-locally free on a Zariski open set $U$ of $X$, and that $\mathscr{X}-U$ is a divisor with normal crossings. Moreover the question is local on $\mathscr{X}$ and the result is true in a neighbourhood of each point of $X=F^{-1}\left(\mathbb{A}^{1}\right)$ $=\mathscr{X}-F^{-1}(\infty)$. So it is enough to prove the result for the $\mathscr{D}[\tau]\left\langle\partial_{\tau}\right\rangle$-module $\mathbf{C}\{x, y, z\}\left[x^{-1}, y^{-1}, \tau\right] \cdot x^{a} y^{b} e^{-\tau / x^{m}}$ where $\left(x_{0}, \cdots, x_{p}, y_{1}, \cdots, y_{q}, z_{1}, \cdots, z_{r}\right)(p \geq 0)$ are local coordinates on $\mathbf{C}^{n+1}, m=\left(m_{0}, \cdots, m_{p}\right) \in(\mathbf{N}-\{0\})^{p+1}, a=\left(a_{0}, \cdots, a_{p}\right) \in \mathbf{C}^{p+1}$, $b=\left(b_{1}, \cdots, b_{q}\right) \in \mathbf{C}^{q}, p+q+r=n$ and $\mathscr{D}=\mathbf{C}\{x, y, z\}\left\langle\partial_{x}, \partial_{y}, \partial_{z}\right\rangle$ denotes the ring of germs of differential operators on $\left(\mathbf{C}^{n+1}, 0\right)$. Moreover, the strong regularity being a local analytic property and depending only on the localized module along $\tau=0$, it is enough to consider $\mathbf{C}\{x, y, z, \tau\}\left[x^{-1}, y^{-1}, \tau^{-1}\right] x^{a} y^{b} \cdot e^{-\tau / x^{m}}$. We shall now forget the coordinates $y, z$ and put $p=n$ (it is easy to reduce to this situation). After a sequence of blowing-up of $\mathbb{C}^{n+2}$, we are reduced to prove the following: if $z=\left(z^{\prime}, z^{\prime \prime}\right)$ are coordinates on $\mathbf{C}^{n+2}$, then $\mathbf{C}\{z\}\left[z^{-1}\right]$. $z^{c} e^{-1 / z^{\prime} \mu^{\prime}}$ is strongly regular along $\left\{f\left(z^{\prime \prime}\right)=0\right\}$ where $f$ is any monomial in the variables $z^{\prime \prime}$. In this situation, the holonomic module is the external product of a strongly regular holonomic module (in the variables $z^{\prime \prime}$ ) along $f^{-1}(0)$ with a holonomic module in the variables $z^{\prime}$. It is not difficult to conclude in this case.

\section{§4. Fourier Transform and Hodge Filtration}

We now come back to the situation considered in the introduction, namely a regular function $f: U \rightarrow \mathbf{A}^{1}$ on a smooth quasi-projective variety $U$. We fix a projective compactification $\mathscr{X}$ of $U$ such that

(1) there exists a rational function $F: \mathscr{X} \rightarrow \mathbb{P}^{1}$ extending $f$,

(2) $\mathscr{X}-U=D \cup F^{-1}(\infty)$ is a divisor with normal crossings.

We keep notation of $\S 1.5$. Remark that $\boldsymbol{R j}_{*}{ }^{p} \mathbf{Q}_{U}$ is a perverse sheaf on $X$ and $j_{+} \mathcal{O}_{U}=\mathcal{O}_{X}[* D]$ is a single holonomic $\mathscr{D}_{X}$-module

Fourier transform for $j_{+} \mathcal{O}_{U}$. Let us summarize the results of the previous sections for $j_{+} \mathcal{O}_{U}$. Recall that $i_{F}$ denotes the graph embedding of $F$. In the following, we forget for simplicity the symbol $i_{F+}$ or $\boldsymbol{R} i_{F *}$ and we use ${ }^{p} \psi_{1 / t}$ instead of ${ }^{p} \psi_{1 / F}$. We have constructed an isomorphism in $\operatorname{Perv}\left(\mathbf{Q}_{X}\right)$ compatible with the monodromy action

$$
{ }^{p} \phi_{\tau} \widetilde{\mho}_{F}\left(R j_{*}^{p} \mathbb{Q}_{U}\right) \stackrel{\sim}{\rightarrow}{ }^{p} \psi_{1 / t}\left(\boldsymbol{R} \kappa_{*}{ }^{p} \mathbb{Q}_{U}\right)
$$


where we view the RHS as an object on $\mathscr{X}$ after taking its direct image by the inclusion $F^{-1}(\infty) \subseteq \mathscr{X}$. This isomorphism is strict with respect to the filtrations

$$
\begin{gathered}
W_{\bullet}{ }^{p} \psi_{1 / t}\left(\boldsymbol{R} \kappa_{*}{ }^{p} \mathbf{Q}_{U}\right) \stackrel{\text { def }}{=}{ }^{p} \psi_{1 / t}\left(\boldsymbol{R} k_{F *} W \cdot \boldsymbol{R j}_{*}{ }^{p} \mathbf{Q}_{U}\right) \\
W_{\bullet}{ }^{p} \phi_{\tau} \mathfrak{\mho}_{F}\left(\boldsymbol{R} j_{*}{ }^{p} \mathbf{Q}_{U}\right) \stackrel{\text { def }}{=}{ }^{p} \phi_{\tau} \widetilde{\mho}_{F}\left(W_{\bullet} \boldsymbol{R} j_{*}{ }^{p} \mathbf{Q}_{U}\right)
\end{gathered}
$$

where $W \cdot \boldsymbol{R}_{*}{ }^{p} \mathbf{Q}_{U}$ is the weight filtration of $\boldsymbol{R}_{*}{ }^{p} \mathbf{Q}_{U}$ in $\operatorname{Perv}\left(\mathbf{Q}_{x}\right)$ (recall that ${ }^{p} \psi_{1 / t}$ and ${ }^{p} \phi_{\tau} \widetilde{\mho}_{F}$ are exact functors from $\operatorname{Perv}\left(\mathbf{Q}_{X}\right)$ to $\operatorname{Perv}\left(\mathbf{Q}_{X}\right)$ ).

We also have a diagram of isomorphisms in $\operatorname{Perv}\left(\mathbf{C}_{\mathscr{X}}\right)$

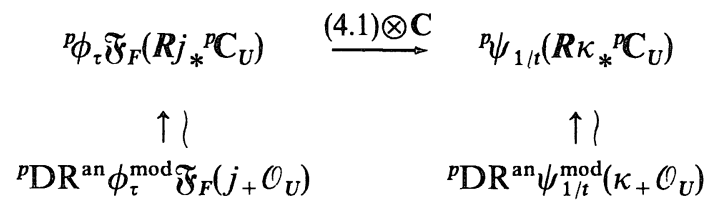

where the left vertical arrow is given by Theorems 2.2 and 3.3 and the right one by Theorem 3.3. By construction, the isomorphism ${ }^{p} \mathrm{DR}^{\text {an }} \phi_{\tau}^{\bmod } \mathfrak{F}_{F}\left(j_{+} \mathscr{O}_{U}\right)$ $\simeq{ }^{p} \mathrm{DR}^{\mathrm{an}} \psi_{1 / t}^{\mathrm{mod}}\left(\kappa_{+} \mathcal{O}_{U}\right)$ that we get is compatible with the $\mathbf{Q}$-structures of both terms.

According to the Riemann-Hilbert correspondence saying that ${ }^{p} \mathrm{DR}^{\text {an }}$ is an equivalence between $\operatorname{Mod}_{\mathrm{hr}}\left(\mathscr{D}_{\mathscr{X}}\right)$ and $\operatorname{Perv}\left(\mathbf{C}_{\mathscr{X}}\right)$, we get an isomorphism

$$
\phi_{\tau}^{\bmod } \mathfrak{F}_{F}\left(j_{+} \mathcal{O}_{U}\right) \simeq \psi_{1 / t}^{\bmod }\left(\kappa_{+} \mathcal{O}_{U}\right)
$$

which is compatible with the monodromy action, so for any $\lambda \in \mathbb{C}^{*}$ we have an isomorphism

$$
\phi_{\tau, \lambda}^{\bmod } \mathfrak{F}_{F}\left(j_{+} \mathcal{O}_{U}\right) \simeq \psi_{1 / t, \lambda}^{\bmod }\left(\kappa_{+} \mathcal{O}_{U}\right)
$$

compatible with the action of the nilpotent endomorphism $N$ defined in $\S 3.1$.

We still denote $W_{\bullet}$ the filtration induced by $W_{\bullet} j_{+} \mathcal{C}_{U}$ on $\kappa_{+} \mathcal{O}_{U}, \psi_{1 / t}^{\bmod }\left(\kappa_{+} \mathcal{O}_{U}\right)$, $\psi_{\tau, \lambda}^{\bmod } \mathfrak{F}_{F}\left(j_{+} \mathcal{O}_{U}\right)$ and $\phi_{\tau, 1}^{\bmod } \widetilde{F}_{F}\left(j_{+} \mathcal{O}_{U}\right)$. By exactness, we have $W \cdot \psi_{1 / t}^{\bmod }\left(\kappa_{+} \mathcal{O}_{U}\right)$ $=\psi_{1 / t}^{\bmod }\left(W_{\bullet} \kappa_{+} \mathcal{O}_{U}\right)$, etc., and (4.2) is strictly filtered with respect to $W_{\circ}$.

By [24] the relative monodromy filtration $M_{\bullet}\left(N, W_{\bullet}\right)$ exists for $N: \psi_{1 / t}^{\bmod } \kappa_{+} \mathcal{O}_{U}$ $\rightarrow \psi_{1 / t}^{\text {mod }} \kappa_{+} \mathcal{O}_{U}$ so it does exist for $N: \phi_{\tau}^{\bmod } \mathfrak{F}_{F}\left(j_{+} \mathcal{O}_{U}\right) \rightarrow \phi_{\tau}^{\text {mod }} \mathfrak{F}_{F}\left(j_{+} \mathcal{O}_{U}\right)$.

Analogous results hold for $\psi_{\tau, 1}^{\text {mod }} \tilde{\mho}_{F}\left(j_{+} \mathcal{O}_{U}\right)$ using the Beilinson functor $\Xi_{F}$ 
and $[24, \S 2 . \mathrm{e}]$ instead of $\psi_{1 / t}^{\mathrm{mod}}$.

Filtrations. Denote $\mathscr{W}_{{ }_{0}} \kappa_{+} \mathcal{O}_{U}$ the weight filtration of $\kappa_{+} \mathcal{O}_{U}=\mathcal{O}_{\mathscr{X}}[*(\mathscr{X}-U)]$ and consider the increasing filtration $F_{\bullet}$ by the order of the pole (functions with a pole of order $\leq 1$ are in $F_{0}$ and $F_{-1}=0$ ). This is a filtration by $\mathcal{O}_{X}$-coherent submodules. Denote $F_{0}^{H} \kappa_{+} \mathcal{O}_{U} \stackrel{\text { def }}{=} F_{0}\left[-d_{U}\right] \kappa_{+} \mathcal{O}_{U}$ the Hodge filtration, with $d_{U}=\operatorname{dim} U$. We will also put $n=d_{U}-1$.

We will also need the filtration $F_{0}^{\prime}$ counting only the order of the pole along $D$ : this is a filtration by $\mathcal{O}_{X}\left[{ }_{*} F^{-1}(\infty)\right]$-coherent submodules.

The $\mathscr{D}_{\mathscr{C} \times \mathbf{A}^{1}}$-module $i_{F+} \kappa_{+} \mathscr{O}_{U}$ comes also equipped with an increasing filtration $F_{0}$ in a natural way. Let $V_{0} i_{F+} \kappa_{+} \mathcal{O}_{U}$ be the Malgrange-Kashiwara filtration along $\mathscr{X} \times\{\infty\}$. Then $F_{0} i_{F+} \kappa_{+} \mathcal{O}_{U}$ induces on each $\operatorname{gr}_{\alpha}^{V} i_{F+} \kappa_{+} \mathcal{O}_{U}$ $\left(\alpha \in\left[0,1[)\right.\right.$ an increasing filtration $F_{\bullet} \psi_{1 / t, \lambda}^{\bmod }\left(\kappa_{+} \mathcal{O}_{U}\right)$ with $\lambda=\exp (2 i \pi \alpha)$. There we put $F_{\bullet}^{H}=F_{\cdot}[-n]$. In the same way the module $\Xi_{F}\left(\kappa_{+} \mathcal{O}_{U}\right)$ comes equipped with a Hodge filtration $F_{0}^{H} \Xi_{F}\left(\kappa_{+} \mathcal{O}_{U}\right)$ (see $[24, \S 2 . \mathrm{e}]$ ).

On the other hand, let $G_{\bullet} \mathscr{D}_{\mathscr{x}}[\tau]\left\langle\partial_{\tau}\right\rangle$ be the increasing filtration by "degree of operators in $\mathscr{D}_{\mathscr{X}}+$ degree in $\tau$ ", so that $G_{0} \mathscr{D}_{\mathscr{X}}[\tau]\left\langle\partial_{\tau}\right\rangle=\mathcal{O}_{X}\left[\partial_{\tau}\right]$.

We put

$$
G_{p} \kappa_{+} \mathscr{E}^{-\tau f^{\text {def }}}=\sum_{q+r \leq p} G_{q} \mathscr{D}_{\mathscr{X}}[\tau]\left\langle\partial_{\tau}\right\rangle \cdot\left(F_{r}^{\prime} \mathcal{O}_{\mathscr{X}}[*(\mathscr{X}-U)] e^{-\tau f}\right) .
$$

This filtration induces on each $\operatorname{gr}_{\alpha}^{V}\left(\kappa_{+} \mathscr{E}^{-\tau f}\right)(\alpha \in[0,1])$ a filtration denoted $\left.\left.G \bullet \phi_{\tau, \lambda}^{\bmod } \mathfrak{F}_{F}\left(j_{+} \mathcal{O}_{U}\right)(\lambda=\exp (2 i \pi \alpha), \alpha \in] 0,1\right]\right)$ or $G \cdot \psi_{\tau, 1}^{\bmod } \mathfrak{F}_{F}\left(j_{+} \mathcal{O}_{U}\right)(\alpha=0)$.

(4.3) Theorem. We have isomorphisms compatible with the monodromy action

$$
\begin{aligned}
& {\left[\psi_{1 / t}^{\bmod }\left(\kappa_{+} \mathcal{O}_{U}\right), F_{\bullet}^{H}, M \cdot(N, W)[n],{ }^{p} \psi_{1 / t}\left(\boldsymbol{R} \kappa_{*}{ }^{p} \mathbf{Q}_{U}\right)\right]} \\
& \simeq\left[\phi_{\tau}^{\mathrm{mod}} \mathfrak{F}_{F}\left(j_{+} \mathcal{O}_{U}\right), G_{\bullet}[-n], M \cdot(N, W)[n],{ }^{p} \phi_{\tau} \mathfrak{\mho}_{F}\left(\boldsymbol{R} j_{*}{ }^{p} \mathbf{Q}_{U}\right)\right] \\
& {\left[\Xi_{F}\left(\kappa_{+} \mathcal{O}_{U}\right), F_{\bullet}^{H}, M_{\bullet}(N, W)[n+1], \Xi_{F}\left(\boldsymbol{R}_{*}{ }^{p} \mathbf{Q}_{U}\right)\right]} \\
& \quad \simeq\left[\psi_{\tau, 1}^{\bmod } \mathfrak{F}_{F}\left(j_{+} \mathcal{O}_{U}\right), G_{\bullet}[-(n+1)], M \cdot(N, W)[n+1],{ }^{p} \psi_{\tau, 1} \mathfrak{F}_{F}\left(\boldsymbol{R}_{*}{ }_{*}^{p} \mathbf{Q}_{U}\right)\right]
\end{aligned}
$$

which makes the RHS a mixed Hodge Module, and the following sequence is an exact sequence of mixed Hodge Modules isomorphic to [24, (2.22.1)] for $\mathscr{M}=\kappa_{+} \mathcal{O}_{U}$ : 


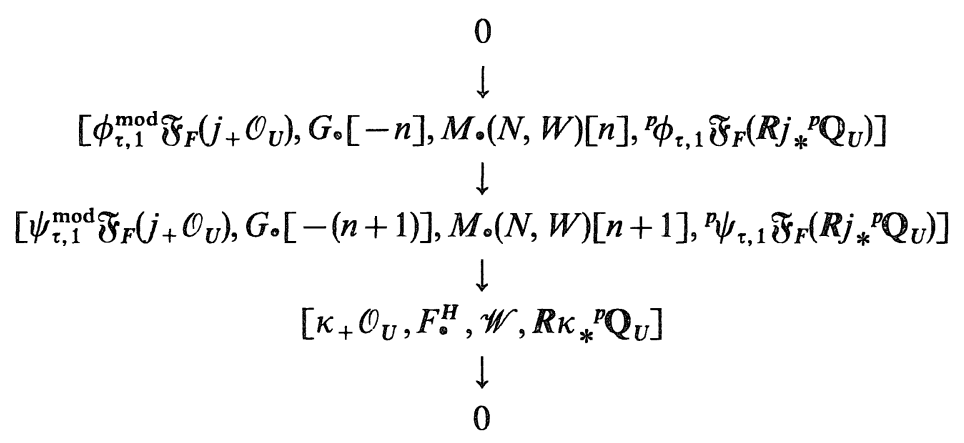

We will give the proof for $\phi_{\tau}^{\text {mod }}$, letting the proof for $\psi_{\tau, 1}^{\text {mod }}$ to the reader. To prove the theorem we will compute locally the $V$-filtration for the modules involved and will show first that there exist locally strict isomorphisms between both terms in (4.2). To show that the isomorphism (4.2) is indeed strict, we will use a trick due to M. Saito [23] (see \$4.21).

Let us analyse the structure of the $V$-filtration of $\kappa_{+} \mathscr{E}^{-\tau f}$ along $\tau=0$ in an analytic neigbourhood of a point on $F^{-1}(\infty)$. So we choose local coordinates $(x, y, z)$ on $\mathscr{X}^{\text {an }}$ compatible with the divisor $D \cup F^{-1}(\infty)$, where $x=\left(x_{0}, \cdots, x_{p}\right)$, $y=\left(y_{1}, \cdots, y_{q}\right), z=\left(z_{1}, \cdots, z_{r}\right)$, such that $p, q, r \geq 0$ and $p+q+r=n$. In these coordinates we have $D=\left\{\Pi_{j=1}^{q} y_{j}=0\right\}$ and we may also assume that $F(x, y, z)=1 / x^{m}$, with $m=\left(m_{0}, \cdots, m_{p}\right)$ and $m_{i}>0$ for all $i=0, \cdots, p$.

We shall denote $\mathscr{D}$ the ring of differential operators with coefficients in $\mathbb{C}\{x, y, z\}$ and $\mathscr{E}$ the free rank one $\mathbb{C}\{x, y, z\}\left[x^{-1}, y^{-1}, \tau\right]$-module generated by $e^{-\tau / x^{m}}$ with its natural structure of a $\mathscr{D}[\tau]\left\langle\partial_{\tau}\right\rangle$-module. We will also use $e=\frac{e^{-\tau / x^{m}}}{x_{0} \cdots x_{n}}$ or $e^{\prime}=\frac{e^{-\tau / x^{m}}}{x_{0} \cdots x_{n} \cdot y_{1} \cdots y_{q}}$ (we put $y_{1} \cdots y_{q}=1$ if $q=0$ ) as a generator of $\mathscr{E}$ as a $\mathbb{C}\{x, y, z\}\left[x^{-1}, y^{-1}, \tau\right]$-module and $e^{\prime}$ as a generator of $\mathscr{E}$ as a $\mathscr{D}[\tau]\left\langle\partial_{\tau}\right\rangle$-module.

Let $G_{\bullet} \mathscr{D}[\tau]\left\langle\partial_{\tau}\right\rangle$ be the increasing filtration by "total degree in $\partial_{x}, \partial_{y}$, $\partial_{z}+$ degree in $\tau$ " and let $G_{\circ} \mathscr{E}$ be the increasing filtration of $\mathscr{E}$ induced by the filtration of $\mathbb{C}\{x, y, z\}\left[x^{-1}, y^{-1}, \tau\right]$ by "total pole order in $y+$ degree in $\tau$ " (here, the pole order is taken in the sense of $[6, \S 3.1 .10]$, i.e. with a shift). For a multiinteger $b$ we will denote $|b|=\Sigma_{j} \max \left(b_{j}-1,0\right)$. Hence

$$
G_{-1} \mathscr{E}=\{0\}, \quad G_{0} \mathscr{E}=\mathbb{C}\{x, y, z\}\left[x^{-1}\right] \cdot e^{\prime},
$$


and more generally, for $k \geq 0$,

$$
G_{k} \mathscr{E}=\sum_{\substack{v \in \mathbf{N} q, l, \mathbf{N} \\|v|+l \leq k}} \mathbf{C}\{x, y, z\}\left[x^{-1}\right] \cdot y^{-v} \tau^{l} \cdot e^{-\tau / x^{m}} .
$$

The following is easy to prove:

(4.4) Lemma. We have $G_{\bullet} \mathscr{E}=G_{\bullet} \mathscr{D}[\tau]\left\langle\partial_{\tau}\right\rangle \cdot e^{\prime}$.

Hence $G_{\bullet} \mathscr{E}$ is a $\operatorname{good} G_{\bullet} \mathscr{D}[\tau]\left\langle\partial_{\tau}\right\rangle$-filtration.

We denote $W_{\mathscr{}} \mathscr{E}$ the increasing filtration of $\mathscr{E}$ by the number of polar components along $D$ :

$$
W_{k} \mathscr{E}=\sum_{\boldsymbol{J} \subset\{1, \cdots, q\}, \# J=k} \mathbf{C}\{x, y, z\}\left[x^{-1}, y_{J}^{-1}, \tau\right] \cdot e^{-\tau / x^{m}}
$$

(4.5) Proposition. For all $\alpha \in[0,1]$,

1. the monodromy filtration of $N: \operatorname{gr}_{\alpha}^{V} \mathscr{E} \rightarrow \operatorname{gr}_{\alpha}^{V} \mathscr{E}$ relative to $W \cdot \operatorname{gr}_{\alpha}^{V} \mathscr{E}$ exists and we denote it $M\left(\operatorname{gr}_{\alpha}^{V} \mathscr{E}, N, W\right)$;

2. for all $i, k$ and $l$, the morphism $\operatorname{gr}_{l}^{M} \operatorname{gr}_{k}^{W} \operatorname{gr}_{\alpha}^{V} \mathscr{E} \rightarrow \operatorname{gr}_{l-2 i}^{M} \operatorname{gr}_{k}^{W} \operatorname{gr}_{\alpha}^{V} \mathscr{E}$ induced by $N^{i}$ strictly shifts by $i$ the filtration induced by $G_{\bullet} \mathscr{E}$ on each term.

Remarks.

We denote $W_{\circ} \operatorname{gr}_{\alpha}^{V} \mathscr{E}$ (resp. $G_{\circ} \operatorname{gr}_{\alpha}^{V} \mathscr{E}$ ) the filtration induced by $W_{\circ} \mathscr{E}$ (resp. $G \circ \mathscr{E})$ on $\operatorname{gr}_{\alpha}^{V} \mathscr{E}$, namely $W_{k} \operatorname{gr}_{\alpha}^{V} \mathscr{E}=W_{k} \cap V_{\alpha}(\mathscr{E}) / W_{k} \cap V_{<\alpha}(\mathscr{E})(r e s p .$.$) . Note that$ $V_{\bullet} \mathscr{E}$ induces on $W_{k} \mathscr{E}$ the Malgrange-Kashiwara filtration $V\left(W_{k} \mathscr{E}\right)$.

The filtration induced by $G_{\bullet} \mathscr{E}$ on $\operatorname{gr}_{l}^{M} \operatorname{gr}_{k}^{W} \operatorname{gr}_{\alpha}^{V} \mathscr{E}$ is the filtration successively induced on each graded term.

The definition and the properties of the relative monodromy filtration are given in [26]. Recall that $N$ shifts it by -2 and that for each $i \geq 0$ the induced morphism $N^{i}: \mathrm{gr}_{k+i}^{M} \mathrm{gr}_{k}^{W} \rightarrow \mathrm{gr}_{k-i}^{M} \mathrm{gr}_{k}^{W}$ is an isomorphism.

Part one of Proposition 4.5 is in fact a consequence of [26] or [24] if one uses the isomorphism (4.1) for $\alpha \in] 0,1]$ and its analogue with $\Xi_{F}$ for $\alpha=0$. The computation that we need to do will show it also.

Computation of the $V$-filtration. In order to prove Proposition 4.5, we shall give an explicit presentation of $\operatorname{gr}_{\alpha}^{V} \mathscr{E}$. 
(4.6) Lemma. Let $e^{\prime}$ as above. Then $e^{\prime}$ is a generator of $\mathscr{E}$ as a $\mathscr{D}[\tau]\left\langle\partial_{\tau}\right\rangle$-module, which satisfies the following equations:

$$
\begin{gathered}
\tau \partial_{\tau} e^{\prime}=-\frac{\tau}{x^{m}} e^{\prime} \\
\partial_{x_{i}} e^{\prime}=-\frac{m_{i}}{x_{i}}\left(\tau \partial_{\tau}+\frac{1}{m_{i}}\right) \cdot e^{\prime} \quad i=0, \cdots, p \\
\partial_{y_{j}} e^{\prime}=-\frac{e^{\prime}}{y_{j}} \quad j=1, \cdots, q \\
\partial_{z_{k}} e^{\prime}=0 \quad k=1, \cdots, r \\
\tau\left(\prod_{i=0}^{p} \partial_{x_{i}}^{m_{i}}\right) \cdot e^{\prime}=-(-m)^{m}\left(\prod_{i=0}^{p} \prod_{k=1}^{m_{\tau}}\left[\tau \partial_{\tau}-1+\frac{k}{m_{i}}\right]\right) \cdot \tau \partial_{\tau} \cdot e^{\prime} .
\end{gathered}
$$

Consequently the order of $e^{\prime}$ with respect to the $V$-filtration of $\mathscr{E}$ is $\leq 0$, so we have $V_{0}\left(\mathscr{D}[\tau]\left\langle\partial_{\tau}\right\rangle\right) \cdot e^{\prime} \subset V_{0}(\mathscr{E})$.

We shall now introduce a family of polynomials of one variable $s$ indexed by a rational number $\alpha$ and a multiindex $a=\left(a_{0}, \cdots, a_{p}\right) \in \mathbb{Z}^{p+1}$. For $\alpha \in \mathbb{Q}$ we put

$$
P_{a, \alpha}(s)=c_{a, \alpha} \prod_{i=0}^{p} \prod_{\left[m_{\imath} \alpha\right]<l \leq a_{\imath}}\left(s+\frac{l}{m_{i}}\right)
$$

where $l$ is an integer, $\left[m_{i} \alpha\right]$ denotes largest integer $\leq m_{i} \alpha$ and we take the convention that the product indexed by the empty set is 1 . The constant $c_{a . \alpha}$ is chosen so that $P_{a, \alpha}(-\alpha)=1$.

For $a \in \mathbb{Z}^{p+1}$ and $\alpha \in \mathbf{Q}$, put $P_{a,<\alpha}(s)=P_{a, \beta}(s)$ for $\beta<\alpha$ and $\beta$ close to $\alpha$. Let $\left\lceil m_{i} \alpha\right\rceil \stackrel{\text { def }}{=}-\left[-m_{i} \alpha\right]$ be the smallest integer bigger than or equal to $m_{i} \alpha$. We hence have

$$
P_{a,<\alpha}(s)=\star \prod_{i=0}^{p} \prod_{\left[m_{i} \alpha\right] \leq l \leq a_{i}}\left(s+\frac{l}{m_{i}}\right)
$$

where $\star$ is a nonzero constant. There exists $\delta_{a, \alpha} \in \mathbf{N}$ and a nonzero constant $\star$ such that $P_{a,<\alpha}(s)=\star(s+\alpha)^{\delta_{a, \alpha}} P_{a, \alpha}(s)$. We have

$$
\delta_{a, \alpha}=\#\left\{i \mid m_{i} \alpha \in \mathbb{Z} \text { and } a_{i} \geq m_{i} \alpha\right\}
$$


hence we have $\delta_{a, \alpha} \leq p+1 \leq n+1$.

We define a correction term $Q_{\alpha}(s)$ by

$$
Q_{\alpha}(s)= \begin{cases}1 & \text { if } \alpha \geq 0 \\ s(s-1) \cdots(s-k+1) & \text { if } k \in \mathbf{N}^{*} \text { and } \alpha \in[-k,-k+1[.\end{cases}
$$

The following properties are easily verified:

\section{(4.7) Lemma.}

1. The family $\left(P_{a, \alpha} Q_{\alpha}\right)$ of ideals of $\mathbf{C}[s]$ is increasing (with respect to the usual partial ordering of $\left.(-\mathbf{Z})^{p+1} \times \mathbf{Q}\right)$, i.e. $P_{a, \alpha} Q_{\alpha}$ is a multiple of $P_{a^{\prime}, \alpha^{\prime}} Q_{\alpha^{\prime}}$ if $-a_{i} \leq-a_{i}^{\prime}$ for all $i$ and $\alpha \leq \alpha^{\prime}$.

2. $P_{a, \alpha}(s) Q_{\alpha}(s)=q_{\alpha}(s) \cdot P_{a+m, \alpha+1}(s-1) Q_{\alpha+1}(s-1)$ where $q_{\alpha}$ is a constant if $\alpha \geq 0$ and $q_{\alpha}(s)=$ const $\cdot s$ (const $\in \mathbf{Q}^{*}$ ) if $\alpha<0$.

3. $\operatorname{gcd}\left(P_{a, \alpha}(s) Q_{\alpha}(s), P_{a-m, \alpha}(s+1) Q_{\alpha}(s+1)\right)=P_{a, \alpha+1}(s) Q_{\alpha+1}(s)$.

In order to compute the $V$-filtration, we shall use another presentation of $\mathscr{E}$ :

(4.8) Lemma. $\mathscr{E}$ is the $\mathbf{C}\{x, y, z\}\left[x^{-1}, y^{-1}, \tau \partial_{\tau}\right]$-module generated by $e^{-\tau / x^{m}}$ and, as such, is free of rank one. The filtration $G_{\bullet} \mathscr{E}$ is the filtration by "total pole order in $y+$ degree in $\tau \partial_{\tau}$ ".

Proof. One considers the map $\mathbf{C}\{x, y, z\}\left[x^{-1}, y^{-1}, \tau \partial_{\tau}\right] \rightarrow \mathbf{C}\{x, y, z\}\left[x^{-1}\right.$, $\left.y^{-1}, \tau\right]$ defined by

$$
\sum f_{k}(x, y, z)\left(\tau \partial_{\tau}\right) \cdots\left(\tau \partial_{\tau}-k+1\right) \mapsto \sum f_{k}(x, y, z)\left(-\frac{\tau}{x^{m}}\right)^{k}
$$

One verifies that this is an isomorphism of $\mathbf{C}\{x, y, z\}\left[x^{-1}, y^{-1}\right]$-modules, which gives the result.

(4.9) Lemma. For $\alpha \in \mathbf{Q}$, let

$$
U_{\alpha} \mathscr{E}=\sum_{a \in \mathbf{Z}^{p+1}} \mathbf{C}\{x, y, z\}\left[y^{-1}, \tau \partial_{\tau}\right] \cdot x^{-a} P_{a, \alpha}\left(\tau \partial_{\tau}\right) Q_{\alpha}\left(\tau \partial_{\tau}\right) \cdot e^{\prime}
$$

Then $U \cdot \mathscr{E}$ is equal to the Malgrange-Kashiwara filtration $V_{\bullet} \mathscr{E}$. 
Remark. We can replace $e^{\prime}$ with $e=y_{1} \cdots y_{q} e^{\prime}$ in the previous expression of $U_{\alpha}$. Using the fact that $e^{-\tau / x^{m}}=x_{0} \cdots x_{m} \cdot e$, the previous expression can be written

$$
V_{\alpha} \mathscr{E}=\sum_{a \in \mathbb{Z}^{p+1}} \mathbb{C}\{x, y, z\}\left[y^{-1}, \tau \partial_{\tau}\right] \cdot x^{-a} P_{a-1, \alpha}\left(\tau \partial_{\tau}\right) Q_{\alpha}\left(\tau \partial_{\tau}\right) \cdot e^{-\tau / x^{m}}
$$

Proof. Remark first that, thanks to Lemma $4.7, U_{\circ} \mathscr{E}$ is an increasing filtration which is in fact discretely indexed, $\tau \cdot U_{\alpha} \subset U_{\alpha-1}$ with equality for $\alpha<0$ (this follows from 4.7-(2)), and $U_{\alpha}+\partial_{\tau} U_{\alpha}=U_{\alpha+1}(4.7-(3))$. Let us show that each $U_{\alpha}$ is stable under the action of $V_{0} \mathscr{D}[\tau]\left\langle\partial_{\tau}\right\rangle=\mathscr{D}[\tau]\left\langle\tau \partial_{\tau}\right\rangle$. The only point which is not completely evident is the stability under $\partial_{x_{i}}$. We have

$$
\begin{aligned}
\partial_{x_{i}}\left(x^{-a} P_{a, \alpha}\left(\tau \partial_{\tau}\right) Q_{\alpha}\left(\tau \partial_{\tau}\right) \cdot e^{\prime}\right) & =-x^{-a-\mathbb{1}_{1}}\left(m_{i} \tau \partial_{\tau}+a_{i}+1\right) P_{a, \alpha}\left(\tau \partial_{\tau}\right) Q_{\alpha}\left(\tau \partial_{\tau}\right) \cdot e^{\prime} \\
& =\left(m_{i} \alpha-a_{i}-1\right) x^{-a-\mathbb{1}_{i}} P_{a+\mathbb{H}_{i}, \alpha}\left(\tau \partial_{\tau}\right) Q_{\alpha}\left(\tau \partial_{\tau}\right) \cdot e^{\prime}
\end{aligned}
$$

and the last term is clearly in $U_{\alpha} \mathscr{E}$.

There exists $\delta_{\alpha}$ such that $\left(\tau \partial_{\tau}+\alpha\right)^{\delta_{\alpha}} \cdot U_{\alpha} \subset U_{<\alpha}$ and we may choose $\delta_{\alpha} \leq n+1$ for $\alpha \notin-\mathbb{N}$ and $\delta_{\alpha} \leq n+2$ for $\alpha \in-\mathbb{N}$.

It is then enough to show that some $U_{\alpha}$ is of finite type over $\mathscr{D}[\tau]\left\langle\tau \partial_{\tau}\right\rangle$ to conclude that $U_{\circ} \mathscr{E}$ is a good filtration which moreover satisfies the same properties than $V_{\bullet} \mathscr{E}$ does, hence both filtrations coincide. But a simple computation shows that $U_{0}=V_{0} \mathscr{D}[\tau]\left\langle\partial_{\tau}\right\rangle \cdot e^{\prime}$.

Proof of Proposition 4.5. Put $\lceil m \alpha\rceil=-[-m \alpha]$. For $a \in \mathbb{Z}^{p+1}$ such that $a \geq\lceil m \alpha\rceil$ (i.e. $a_{i} \geq\left\lceil m_{i} \alpha\right\rceil$ for all $i$ ) we put $I_{\alpha}(a)=\left\{i \mid a_{i}=\left\lceil m_{i} \alpha\right\rceil\right\}$ and $x_{I_{\alpha}(a)}=\left(x_{i}\right)_{i \in I_{\alpha}(a)}$. For $b \in \mathbb{N}^{q}$ put $J(b)=\left\{j \mid b_{j}=0\right\}$ and define $y_{J(b)}$ in an analogous way.

For $\alpha \in[0,1]$, it will be convenient to denote

$$
\delta_{a, \alpha}(+1)= \begin{cases}\delta_{a, \alpha} & \text { if } \alpha \in] 0,1] \\ \delta_{a, \alpha}+1 & \text { if } \alpha=0 .\end{cases}
$$

(4.11) Lemma。 Let $\alpha \in[0,1]$.

1. Every element $\varepsilon \in V_{\alpha} \mathscr{E}$ can be written in a unique way

$$
\left[\sum_{a \geq\lceil m \alpha]} \sum_{b \geq 0} \sum_{\lambda \geq 0} g_{a, b, \lambda}\left(x_{I_{\alpha}(a)}, y_{J(b)}, z\right) \cdot x^{-a} y^{-b} \cdot\left(\tau \partial_{\tau}+\alpha\right)^{\lambda} P_{a-1, \alpha}\left(\tau \partial_{\tau}\right)\right] \cdot e^{-\tau / x^{m}}
$$


where, for all $a, b, \lambda, g_{a, b, \lambda} \in \mathbb{C}\left\{x_{I(a)}, y_{J(b)}, z\right\}$, and $g_{a, b, \lambda} \equiv 0$ or $g_{a, b, \lambda}(0,0, z)$ $\neq 0$.

2. We have $\varepsilon \in V_{<\alpha} \mathscr{E}$ if and only if $\left(g_{a, b, \lambda} \neq 0 \Rightarrow \lambda \geq \delta_{a-1, \alpha}(+1)\right)$.

3. We have

$$
\begin{aligned}
\operatorname{ord}_{G} \varepsilon & =\max _{\left\{(a, b, \lambda) \mid g_{a, b, \lambda} \neq 0\right\}}|b|+\lambda+\operatorname{deg} P_{a-1, \alpha} \\
\operatorname{ord}_{W} \varepsilon & =\max _{\left\{(a, b, \lambda) \mid g_{a, b, \lambda} \neq 0\right\}} \# J(b) .
\end{aligned}
$$

Proof. We consider on the set of tuples $\mathbb{Z}^{p+1} \times \mathbb{Z}^{q} \times \mathbb{N}$ the lexicographic order. This allows one to define on $\mathbb{C}\{x, y, z\}\left[x^{-1}, y^{-1}, \tau \partial_{\tau}+\alpha\right]$ (hence on $\mathscr{E}$ ) a filtration $\mathscr{F}$ indexed by $\mathbb{Z}^{p+1} \times \mathbb{Z}^{q} \times \mathbb{N}$ : an element $\phi$ has order $(a, b, d)$ if $a_{0}$ is the pole order along $x_{0}=0$, then $a_{1}$ is the pole order along $x_{1}=0$ of the coefficient of $x^{-a_{0}}$, etc. and $d$ is the degree with respect to $\tau \partial_{\tau}+\alpha$.

Remark also that we have $P_{\max (a-1,[m \alpha]-1), \alpha}=P_{a-1, \alpha}$. Hence each element of $V_{\alpha} \mathscr{E}$ can be written

$$
\varepsilon=\left[\sum_{a \geq\lceil m \alpha]} h_{a}\left(x, y, y^{-1}, z, \tau \partial_{\tau}+\alpha\right) x^{-a} P_{a-1, \alpha}\left(\tau \partial_{\tau}\right)\right] \cdot e^{-\tau / x^{m}}
$$

and if we decompose the $h_{a}$ we obtain an expression

$$
\varepsilon=\left[\sum_{a \geq\lceil m \alpha]} \sum_{b \geq 0} h_{a, b}\left(x, y, z, \tau \partial_{\tau}+\alpha\right) x^{-a} y^{-b} P_{a-1 . \alpha}\left(\tau \partial_{\tau}\right)\right] \cdot e^{-\tau / x^{m}}
$$

with $h_{a, b} \in \mathbf{C}\{x, y, z\}\left[\tau \partial_{\tau}+\alpha\right]$. We will show that the dominant term $\varepsilon_{(a(\varepsilon), b(\varepsilon), d(\varepsilon))}$ of $\varepsilon$ with respect to $\mathscr{F}$ can be written

$$
g_{(a(\varepsilon), b(\varepsilon), \lambda(\varepsilon))} x^{-a(\varepsilon)} y^{-b(\varepsilon)}\left(\tau \partial_{\tau}+\alpha\right)^{\lambda(\varepsilon)} P_{a(\varepsilon)-\mathbb{1}, \alpha}\left(\tau \partial_{\tau}\right) \cdot e^{-\tau / x^{m}}
$$

with $d(\varepsilon) \in \lambda(\varepsilon)+\operatorname{deg} P_{a(\varepsilon)-\mathbb{1}, \alpha}$, so in particular is in $V_{\alpha} \mathscr{E}$. In other words, we will have $\varepsilon \in V_{\alpha} \mathscr{E}$ if and only if this is so for $\varepsilon_{(a(\varepsilon), b(\varepsilon) d(\varepsilon))}$ and $\varepsilon-\varepsilon_{(a(\varepsilon), b(\varepsilon), d(\varepsilon))}$.

For this, consider first term of highest pole order $a_{0}(\varepsilon)$ along $x_{0}=0$. It is also the dominant term of the previous sum indexed by the $(a, b)$ such that $a_{0} \geq a_{0}(\varepsilon)$. This sum can hence be written $H\left(x_{0}, x^{\prime}, y, z, \tau \partial_{\tau}+\alpha\right)$ $\cdot x_{0}^{-a_{0}(\varepsilon)} P_{a_{0}(\varepsilon)-1, \alpha}\left(\tau \partial_{\tau}\right) \cdot e^{-\tau / x^{m}}$ with $x^{\prime}=\left(x_{1}, \cdots, x_{p}\right)$ and $P_{a_{0}-1 . \alpha}=c_{a_{0}-1, \alpha} \prod_{\left[m_{0} \alpha\right]<l \leq a_{0}-1}$ $\left(\tau \partial_{\tau}+l / m_{0}\right)$. Moreover, $H$ admits a similar expression with one variable less 
and we have $H\left(0, x^{\prime}, y, z, \tau \partial_{\tau}+\alpha\right) \neq 0$ so that in this case we may replace $H$ with $H\left(0, x^{\prime}, y, z, \tau \partial_{\tau}+\alpha\right) \neq 0$ in order to compute the dominant term. We then get the result by induction on the number of variables $x$ and $y$.

We deduce from this fact the existence and uniqueness of the decomposition 4.11-(1), as well as the fact that $\varepsilon \in V_{<\alpha} \mathscr{E}$ if and only if $\lambda \geq \delta_{a-1, \alpha}(+1)$ if $g_{a, b, \lambda} \neq 0$ (i.e. 4.11-(2)).

For $\varepsilon \in V_{\alpha} \mathscr{E}$ we have at our disposal two Newton polyhedra: the first one $N(\varepsilon)$ is attached to any $\varepsilon \in \mathscr{E}$, using the unique decomposition

$$
\varepsilon=\left[\sum_{a \geq\lceil m \alpha 1} \sum_{b \geq 0} \sum_{d \geq 0} h_{a, b, d}\left(x_{I_{\alpha}(a)}, y_{J(b)}, z\right) x^{-a} y^{-b}\left(\tau \partial_{\tau}\right)^{d}\right] \cdot e^{-\tau / x^{m}}
$$

with $h_{a, b, d} \neq 0 \Rightarrow h_{a, b . d}(0,0, z) \neq 0$; then $N(\varepsilon)$ is the convex hull of the points $(a, b, d)-\left(\mathbf{N}^{p+1} \times \mathbf{N}^{q} \times \mathbf{N}\right)$ for which $h_{a, b . d} \neq 0$; the second one $N_{\alpha}(\varepsilon)$ is defined in the same way using the decomposition 4.11-(1) of $\varepsilon$. One shows by induction on $(a, b, d)$ that both polyhedra coincide. The filtrations $G$ and $W$ being defined only in terms of the polyhedron $N$, we see that on $V_{\alpha}$ both filtrations can be defined in terms of $N_{\alpha}$, which gives the third point of the lemma.

We deduce from the lemma that if $[\varepsilon]$ denotes the class of $\varepsilon$ in $\operatorname{gr}_{\alpha}^{V} \mathscr{E}$, then $[\varepsilon]$ has a canonical representative $\bar{\varepsilon}$ defined by the formula

$\left[\sum_{a \geq\lceil m \alpha]} \sum_{b \geq 0} \sum_{0 \leq \lambda<\delta_{a-1, \alpha}(+1)} g_{a, b, \lambda}\left(x_{I_{\alpha}(a)}, y_{J(b)}, z\right) \cdot x^{-a} y^{-b} \cdot\left(\tau \partial_{\tau}+\alpha\right)^{\lambda} P_{a-1, \alpha}\left(\tau \partial_{\tau}\right)\right] \cdot e^{-\tau / x^{m}}$ and that if $[\varepsilon] \neq 0$ we have

$$
\begin{gathered}
\operatorname{ord}_{G}[\varepsilon]=\operatorname{ord}_{G} \bar{\varepsilon}=\underset{\left\{(a, b, \lambda) \mid g_{a, b, \lambda} \neq 0 \text { and } \lambda<\delta_{a-1, \alpha}(+1)\right\}}{\max }|b|+\lambda+\operatorname{deg} P_{a-1, \alpha} \\
\operatorname{ord}_{W}[\varepsilon]=\operatorname{ord}_{W} \bar{\varepsilon}=\max _{\left\{(a, b, \lambda) \mid g_{a, b, \lambda} \neq 0 \text { and } \lambda<\delta_{a-1, \alpha}(+1)\right\}} \# J(b) .
\end{gathered}
$$

Denote $M_{\bullet}^{\prime}=M^{\prime}(N)$. the monodromy filtration of $N=\tau \partial_{\tau}+\alpha$ on $\operatorname{gr}_{\alpha}^{V} \mathscr{E}$ centered at 0 . Then using the fact that the order of $N^{\lambda}$ with respect to the filtration $M_{0}^{\prime} \mathbf{C}[N] / N^{\delta}$ is equal to $\delta-1-2 \lambda$, we have $[\varepsilon] \in M_{l}^{\prime} \operatorname{gr}_{\alpha}^{V} \mathscr{E}$ if and only if the only possible nonzero terms in $\bar{\varepsilon}$ are such that $0 \leq \lambda<\delta_{a-1, a}(+1)$ and 
$\delta_{a-1, \alpha}(+1)-1-2 \lambda \leq l$.

We deduce easily from this remark the following consequences, hence 4.5-(1):

1. For all $l \in \mathbf{N}$, the morphism $N^{l}: \operatorname{gr}_{\alpha}^{V} \mathscr{E} \rightarrow \operatorname{gr}_{\alpha}^{V} \mathscr{E}$ is strict with respect to the filtration $W \cdot \operatorname{gr}_{\alpha}^{V} \mathscr{E}$.

2. The relative monodromy filtration $M_{\bullet}=M_{\bullet}\left(\operatorname{gr}_{\alpha}^{V} \mathscr{E}, N, W\right)$ exists and is equal to the convolution $\left(M_{\bullet}^{\prime} \star W_{\bullet}\right) \operatorname{gr}_{\alpha}^{V} \mathscr{E}$ (see [26, Prop. (2.11)]).

3. We have $\operatorname{gr}_{l}^{M} \operatorname{gr}_{k}^{W} \operatorname{gr}_{\alpha}^{V} \mathscr{E}=\operatorname{gr}_{l-k}^{M^{\prime}} \operatorname{gr}_{k}^{W} \operatorname{gr}_{\alpha}^{V} \mathscr{E}$ (see [26, Cor. (1.6)]).

One verifies in the same way that $\varepsilon \in V_{\alpha} \mathscr{E}$ has a nonzero class in $\operatorname{gr}_{l}^{M} \operatorname{gr}_{k}^{W} \operatorname{gr}_{\alpha}^{V} \mathscr{E}$ if and only if there exists $(a, b, \lambda)$ such that $a \geq\lceil m \alpha\rceil, \# J(b)=k, 0 \leq \lambda<\delta_{a-1, \alpha}(+1)$, $\delta_{a-1, \alpha}(+1)-1-2 \lambda=l-k$ and $g_{a, b, \lambda} \neq 0$. The order of the class of $\varepsilon$ with respect to the filtration induced by $G$ is the order of the canonical representative, namely the max of $|b|+\lambda+\operatorname{deg} P_{a-1, \alpha}$ on such tuples $(a, b, \lambda)$. This gives immediately the second point of 4.5 .

From this description we also deduce

(4.12) Proposition. On $\kappa_{+} \mathscr{E}^{-\tau f}$, the filtrations $V_{\bullet}, W_{\bullet}$ and $G_{0}$ are compatible.

Proof. This means that for all $k, d, \alpha$ we have

$$
W_{k} \cap\left[G_{d} \cap V_{\alpha}+V_{<\alpha}\right] \subset\left[W_{k} \cap G_{d} \cap V_{\alpha}\right]+V_{<\alpha}
$$

and this implies that the possible tri-graded objects obtained by permuting the filtrations are all equal. In order to show (4.13), remark that, for $\varepsilon \in V_{\alpha} \mathscr{E}$, we have $\varepsilon \in G_{d} \cap V_{\alpha}+V_{<\alpha}$ if and only if the canonical representative $\bar{\varepsilon}$ is in $G_{d}$. The same equivalence holds for $W_{k} \cap V_{\alpha}+V_{<\alpha}$, so we see that $\varepsilon \in\left[G_{d} \cap V_{\alpha}+V_{<\alpha}\right]$ $\cap\left[W_{k} \cap V_{\alpha}+V_{<\alpha}\right]$ if and only if $\bar{\varepsilon} \in G_{d} \cap W_{k}$, hence the result.

Computation of the primitive part. Let $\alpha \in[0,1]$ and consider the following filtered $\mathscr{D}_{\mathscr{X}}$-module, denoted

$$
\mathcal{O}_{x}\left[*\left(F^{-1}(\infty) \cup D\right)\right] \cdot " F^{-\alpha} \text { " or also } \mathcal{O}_{x}\left[*\left(F^{-1}(\infty) \cup D\right)\right]_{\alpha} .
$$

This is the free $\mathcal{O}_{x}\left[*\left(F^{-1}(\infty) \cup D\right)\right]$-module of rank one with one generator

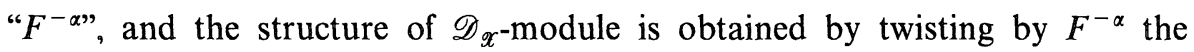
usual one on $\mathcal{O}_{x[}\left[*\left(F^{-1}(\infty) \cup D\right)\right]$. This module comes equipped with the 
following filtrations:

- $W_{0}$ is the filtration by the number of components. of $D$ along which a local section has poles;

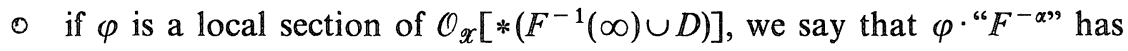
a pole along a component of $F^{-1}(\infty)$ if $F^{-\alpha}$ has an integral multiplicity along this component and if $\varphi F^{-\alpha}$ has a pole along this component; then $\mathscr{W}_{\circ}^{\prime}$ counts the number of components of $\mathbb{F}^{-1}(\infty)$ which are polar for $\varphi^{\prime \prime} F^{-\alpha "}$ and $\mathscr{W}$ 。 is the convolution of $\mathscr{W}^{\prime}$ and $W$;

(4) the filtration $\mathbb{F}_{0}$ is defined by

$$
\operatorname{ord}_{F}\left(\varphi^{“\left(F^{-\alpha}\right)}\right)=\operatorname{ord}_{F}\left(x^{[m \alpha]} \varphi\right)
$$

where the RHS is the pole order in $\mathbb{C}\{x, y, z\}\left[x^{-1}, y^{-1}\right]$ as in $[6, \S 3.1 .10]$.

(4.14) Proposition. For $\alpha \in[0,1]$ we have local filtered isomorphisms

$$
\left(P \operatorname{gr}_{l(+1)}^{M} \operatorname{gr}_{k}^{W} \operatorname{gr}_{\alpha}^{V} \mathscr{E}, G_{0}\right) \stackrel{\sim}{\rightarrow}\left(\operatorname{gr}_{l+1}^{\mathscr{W}} \operatorname{gr}_{k}^{W} \mathcal{O}_{\mathscr{X}}\left[*\left(F^{-1}(\infty) \cup D\right)\right]_{\alpha}, F_{0}\right)
$$

In the $\mathbb{L H S}$, we put $l(+1)=l$ if $\alpha \in] 0,1]$ and $l(+1)=l+1$ if $\alpha=0$.

Proof. We keep notation as above. From 4.5-(2) follows that the filtration $G_{\circ} \operatorname{gr}_{l}^{M} \operatorname{gr}_{k}^{W} \operatorname{gr}_{\alpha}^{V} \mathscr{E}$ is compatible with the Lefschetz decomposition. Assume that $\varepsilon$ has multiorder $(l, k, \alpha)$ with respect to the filtration $M W V$. Then the class of $\varepsilon$ in $\operatorname{gr}_{l}^{M} \operatorname{gr}_{k}^{W} \operatorname{gr}_{\alpha}^{V} \mathscr{E}$ is in the primitive part

$$
P \operatorname{gr}_{l}^{M} \operatorname{gr}_{k}^{W} \operatorname{gr}_{\alpha}^{V} \mathscr{E} \stackrel{\text { def }}{=} \operatorname{Ker} N^{l-k+1}: \operatorname{gr}_{l}^{M} \operatorname{gr}_{k}^{W} \operatorname{gr}_{\alpha}^{V} \mathscr{E} \rightarrow \operatorname{gr}_{2 k-l-2}^{M} \mathrm{gr}_{k}^{W} \operatorname{gr}_{\alpha}^{V} \mathscr{E}
$$

if and only if the canonical representative of this class is

$$
\sum_{\substack{b \geq 0 \\ \sharp J(b)=k}} \sum_{\substack{a \geq I m \alpha] \\ \delta a-1, \alpha(+1)=l-k+1}} g_{a, b, 0}\left(x_{I_{\alpha}(a)}, y_{J(b)}, z\right) x^{-a} y^{-b} P_{a-1, \alpha}\left(\tau \partial_{\tau}\right) \cdot e^{-\tau / x^{m}}
$$

Now, each element $h \in \mathbb{C}\{x, y, z\}\left[x^{-1}, y^{-1}\right]_{\alpha}$ has a unique decomposition

$$
h=\sum_{a \geq[m \alpha]} \sum_{b \geq 0} h_{a, b}\left(x_{I_{\alpha}(a)}, y_{J(b)}, z\right) x^{-a} y^{-b}
$$


with $h_{a, b} \neq 0 \Rightarrow h_{a, b}(0,0, z) \neq 0$. The filtrations on $\mathbb{C}\{x, y, z\}\left[x^{-1}, y^{-1}\right]_{\alpha}$ can be expressed as follows:

- $\operatorname{ord}_{W} h=\max _{h_{a, b} \neq 0} \# J(b)$;

- $\operatorname{ord}_{\mathscr{W}}, h=\max _{h_{a, b} \neq 0}\left(\delta_{a-1, \alpha}\right)$ and $\operatorname{ord}_{w} h=\max _{h_{a, b} \neq 0}\left(\delta_{a-1, \alpha}+\# J(b)\right)$;

- $\operatorname{ord}_{F} h=\max _{h_{a, b} \neq 0}|a-\lceil m \alpha\rceil|+|b|$.

The filtrations $W_{\bullet}$ and $\mathscr{W}_{\bullet}$ are filtrations by $\mathbb{C}\{x, y, z\}\left\langle\partial_{x}, \partial_{y}, \partial_{z}\right\rangle-$ submodules: this is well known for $W_{\bullet}$ so it is enough to verify this for $\mathscr{W}_{\bullet}^{\prime}$, and it is enough to show that if $h_{a, b} x^{-a} y^{-b} \in \mathscr{W}_{l}^{\prime}$ and $h_{a, b}$ satisfies the previous properties, then

$$
\left[\partial_{x_{i}}+m_{i} \alpha / x_{i}\right]\left(h_{a, b} x^{-a} y^{-b}\right)=\partial_{x_{i}}\left(h_{a, b}\right) x^{-a} y^{-b}+\left(m_{i} \alpha-a_{i}\right) h_{a, b} x^{-\left(a+\mathbb{1}_{i}\right)} y^{-b}
$$

is in $\mathscr{W}_{l}^{\prime}$. But we have $\delta_{a+1_{1}-1, \alpha}=\delta_{a-1, \alpha}$ if $a_{i} \neq m_{i} \alpha$, hence each term is in $\mathscr{W}_{l}^{\prime}$.

It is also easy to verify that $F_{0}$ is a good filtration with respect to the usual filtration $F_{\bullet} \mathbb{C}\{x, y, z\}\left\langle\partial_{x}, \partial_{y}, \partial_{z}\right\rangle$ by the total degree in $\partial_{x}, \partial_{y}, \partial_{z}$.

If $h$ has $W$-order $k$ and $\mathscr{W}$-order $l+1$, its class in $\operatorname{gr}_{l+1}^{\mathscr{W}} \operatorname{gr}_{k}^{W} \mathbb{C}\{x, y, z\}\left[x^{-1}\right.$, $\left.y^{-1}\right]_{\alpha}$ is nonzero if and only if the canonical representative

$$
\bar{h}=\sum_{\substack{b \geq 0 \\ \sharp J(b)=k}} \sum_{\substack{a \geq\lceil m \alpha] \\ \delta_{a-1, \alpha}=l-k+1}} h_{a, b}\left(x_{I_{\alpha}(a)}, y_{J(b)}, z\right) x^{-a} y^{-b}
$$

of this class is nonzero. The $F$-order of this class (with respect to the filtration successively induced by $F$ ) is then the $F$-order of the canonical representative $\bar{h}$. Proposition 4.14 will follow from

(4.16) Lemma. Let $\alpha \in[0,1]$. For every $k, l$ the map which sends

$$
\sum_{\substack{b \geq 0 \\ \sharp J(b)=k}} \sum_{\substack{a \geq\lceil m a] \\ \delta_{a-1, \alpha}=l-k+1}} h_{a, b}\left(x_{I_{\alpha}(a)}, y_{J(b)}, z\right) x^{-a} y^{-b}
$$

to

$$
\sum_{\substack{b \geq 0 \\ \sharp J(b)=k}} \sum_{\substack{a \geq[m \alpha] \\ \delta a-1, \alpha=l-k+1}} h_{a, b}\left(x_{I_{\alpha}(a)}, y_{J(b)}, z\right) x^{-a} y^{-b} P_{a-1, \alpha}\left(\tau \partial_{\tau}\right) \cdot e^{-\tau / x^{m}}
$$

induces a strict isomorphism of filtered $\left(\mathbb{C}\{x, y, z\}\left\langle\partial_{x}, \partial_{y}, \partial_{z}\right\rangle, F_{\circ}\right)$-modules 


$$
\left(\mathrm{gr}_{l+1}^{\mathscr{W}} \operatorname{gr}_{k}^{W} \mathbb{C}\{x, y, z\}\left[x^{-1}, y^{-1}\right]_{\alpha}, F_{\bullet}\right) \stackrel{\sim}{\rightarrow}\left(P \operatorname{gr}_{l(+1)}^{M} \operatorname{gr}_{k}^{W} \operatorname{gr}_{\alpha}^{W} \mathscr{E}, G_{\bullet}\right)
$$

Proof. Once $\mathscr{D}$-linearity is proved, bijectivity comes from the uniqueness of the decomposition, and the fact that this isomorphism is strictly filtered comes from the fact that for $a \geq\lceil m \alpha\rceil$, we have $\operatorname{deg} P_{a-1, \alpha}=|a-\lceil m \alpha\rceil|$.

First, one easily see that this mapping is $\mathbf{C}\{z\}$-linear.

The main point to be shown to obtain $\mathbf{C}\{x, y, z\}$-linearity is the fact that if $a \in \mathbb{Z}^{p+1}$ and $b \in \mathbb{N}^{q}$ are fixed, with $a \geq\lceil m \alpha\rceil$, and if $\varphi \in \mathbb{C}\{x, y, z\}$, then the image of the class of $\varphi \cdot x^{-a} y^{-b}$ is the class of $\varphi \cdot x^{-a} y^{-b} P_{a-1, \alpha} \cdot e^{-\tau / x^{m}}$.

We can decompose

$$
\varphi \cdot x^{-a} y^{-b}=\sum_{[m \alpha] \leq a^{\prime} \leq a} \sum_{0 \leq b^{\prime} \leq b} \varphi_{a^{\prime} \cdot b^{\prime}} \cdot x^{-a^{\prime}} y^{-b^{\prime}}
$$

with $\varphi_{a^{\prime}, b^{\prime}} \in \mathbb{C}\left\{x_{I_{\alpha}\left(a^{\prime}\right)}, y_{J\left(b^{\prime}\right)}, z\right\}$ and $\varphi_{a^{\prime}, b^{\prime}} \neq 0 \Rightarrow \varphi_{a^{\prime}, b^{\prime}}(0,0, z) \neq 0$. The image of the class of $\varphi \cdot x^{-a} y^{-b}$ is then equal to the class of

$$
\sum_{[m \alpha] \leq a^{\prime} \leq a} \sum_{0 \leq b^{\prime} \leq b} \varphi_{a^{\prime} b^{\prime}} \cdot x^{-a^{\prime}} y^{-b^{\prime}} P_{a^{\prime}-1, \alpha} \cdot e^{-\tau / x^{m}}
$$

On the other hand we have for $\lceil m \alpha\rceil \leq a^{\prime} \leq a$ the equality

$$
P_{a-1, \alpha}\left(\tau \partial_{\tau}\right)=P_{a^{\prime}-1, \alpha}\left(\tau \partial_{\tau}\right) \cdot q_{a, a^{\prime}}\left(\tau \partial_{\tau}\right)
$$

with $q_{a, a^{\prime}}(-\alpha)=1$. So the class of $\varphi \cdot x^{-a} y^{-b}$ in $\operatorname{gr}_{l(+1)}^{M} \operatorname{gr}_{k}^{W} \operatorname{gr}_{\alpha}^{V} \mathscr{E}$ is equal to

$$
\sum_{[m \alpha] \leq a^{\prime} \leq a} \sum_{0 \leq b^{\prime} \leq b} \varphi_{a^{\prime}, b^{\prime}} \cdot x^{-a^{\prime}} y^{-b^{\prime}} P_{a^{\prime}-1, \alpha} q_{a, a^{\prime}}\left(\tau \partial_{\tau}\right) \cdot e^{-\tau / x^{m}}
$$

and its projection in the primitive part is then equal to (4.17).

$\partial_{x}$-linearity for instance is obtained by comparing (4.15) with a formula analogous to $(4.10)$.

(4.18) Corollary. For $\alpha \in[0,1]$ any local isomorphism of $\mathscr{D}_{\mathscr{X}}$-modules

$$
P \operatorname{gr}_{l(+1)}^{M} \operatorname{gr}_{k}^{W} \operatorname{gr}_{\alpha}^{V} \stackrel{\mathscr{E}}{\stackrel{\sim}{\rightarrow}} \operatorname{gr}_{l+1}^{\mathscr{W}} \operatorname{gr}_{k}^{W} \mathcal{O}_{x}\left[*\left(F^{-1}(\infty) \cup D\right)\right]_{\alpha}
$$

is strictly filtered with respect to the filtrations $G_{\circ}$ and $F_{0}$.

Proof. Let $\{0, \cdots, p\}_{\alpha} \subset\{0, \cdots, p\}$ be the set of indices $i$ such that 
$m_{i} \alpha \in \mathbf{Z}$. For $I \subset\{0, \cdots, p\}_{\alpha}$ with $\sharp I=l+1-k$ and $J \subset\{1, \cdots, q\}$ with $\sharp J=k$, put $E_{I} \cap D_{J}=\left(\cap_{i \in I} E_{i}\right) \cap\left(\cap_{j \in J} D_{j}\right)$. Then the formula above for $\bar{h}$ shows that (at least locally) $\left(\operatorname{gr}_{l+1}^{\mathscr{W}} \operatorname{gr}_{k}^{W} \mathcal{O}_{x}\left[*\left(F^{-1}(\infty) \cup D\right)\right]_{\alpha}, F_{\bullet}\right)$ is equal to the filtered direct sum (over $I, J$ as above) of the largest submodules $M_{I, J}$ supported on $E_{I} \cap D_{J}$, equipped with the induced filtration $F_{\bullet}$.

Any automorphism of $\operatorname{gr}_{l+1}^{W} \operatorname{gr}_{k}^{W} \mathcal{O}_{x}\left[*\left(F^{-1}(\infty) \cup D\right)\right]_{\alpha}$ preserves this decomposition, hence it is enough to verify, thanks to Proposition 4.14, that any automorphism of $M_{I, J}$ is strictly filtered, and showing that any such automorphism is a constant multiple of the identity will be enough.

One can verify that ${ }^{p} \mathrm{DR}^{\text {an }} M_{I . J}$ is equal, up to a shift, to the direct image by the inclusion $E_{I} \cap D_{J} \hookrightarrow \mathscr{X}$ of the following sheaf: on $E_{I}^{o} \cap D_{J} \stackrel{\text { def }}{=} E_{I} \cap D_{J}$ $-\left(\underset{i \notin\{0, \cdots, p\} \alpha}{\cup} E_{i}\right)$, this is the rank one local system with monodromy $\exp 2 i \pi m_{i} \alpha$ around $E_{i} \cap E_{I} \cap D_{J}$ for $i \notin\{0, \cdots, p\}_{\alpha}$; it is extended by 0 on $E_{I} \cap D_{J}$ (this is also the maximal extension). Any automorphism of this sheaf is a constant multiple of identity, so we can conclude using the full faithfulness of ${ }^{p} \mathrm{DR}^{\mathrm{an}}$.

Local comparison with the nearby cycles of $f$ at infinity. The local computation made in [26] for the nearby cycles at infinity (in the case of unipotent monodromy) can be made using arguments as above. We shall sketch it below.

Consider on $\mathscr{X}$ the complex $\boldsymbol{R} \kappa_{*}{ }^{p} \mathbf{Q}_{U}$ and let $\psi_{1 / F}\left(\boldsymbol{R} \kappa_{*}{ }^{p} \mathbf{Q}_{U}\right)$ be the nearby cycle complex on $F^{-1}(\infty)$. It depends only on $R j_{*}{ }^{p} \mathbf{Q}_{U}$ and not on the extension to $\mathscr{X}$ that we have chosen. So we denote it $\psi_{1 / F}\left(\boldsymbol{R} j_{*}{ }^{p} \mathbf{Q}_{U}\right)$.

We denote $t^{\prime}$ the coordinate at infinity on $\mathrm{A}^{1}$. Consider on $\mathscr{X} \times D^{\prime}$, where $D^{\prime}$ is a disc centered at $\infty$ in $\mathbf{P}^{1}$ with coordinate $t^{\prime}$, the $\mathscr{D}_{\mathscr{C} \text { an } \times D^{\prime}}$-module $\mathscr{M}=\left(j_{+} \mathcal{O}_{U}\right)^{\text {an }}\left[\partial_{t^{\prime}}\right] \cdot \delta\left(t^{\prime}-F^{\prime}\right)$ with $F^{\prime}=1 / F$. This is a regular holonomic module on $\mathscr{X}^{\mathrm{an}} \times D^{\prime}$ and ${ }^{p} \mathrm{DR}^{\text {an }} \mathscr{M}$ is the direct image of the sheaf $R j_{*}{ }^{p} \mathbf{C}_{U}$ by the inclusion of the graph of $F^{\prime}$. In the same way, if $i^{\prime}: F^{-1}(\infty) \subseteq \mathscr{X}$ denotes the inclusion, we have $\boldsymbol{R} i_{*}^{\prime} \psi_{1 / F}\left(R j_{*}^{p} \mathbf{C}_{U}\right) \simeq{ }^{p} \operatorname{DR}^{\text {an }} \psi_{t^{\prime}}^{\bmod }(\mathscr{M})$.

This module $\mathscr{M}$ comes equipped with a filtration $F_{0} \mathscr{M}$ good with respect to the usual filtration $F_{0} \mathscr{D}_{\mathscr{X}^{\text {an }} \times D^{\prime}}$ by the order of the operators: this is the filtration of $\left(j_{+} \mathcal{O}_{U}\right)^{\text {an }}\left[\partial_{t^{\prime}}\right] \cdot \delta\left(t^{\prime}-F^{\prime}\right)$ by the degree in $\partial_{t^{\prime}}$. It comes also equipped with a filtration $W$. (number of components of $D$ along which a section has a pole).

The analogue of Proposition 4.5 is true for $\psi_{t^{\prime}}^{\bmod } \mathscr{M}$ : this is proved in [26] 
or [24] (in proving the proposition below we shall recall the proof, which is completely analogous to the one of 4.5 ).

(4.19) Proposition. For all $\alpha \in[0,1[$ and $k, l \in \mathbb{Z}$ we have local filtered isomorphisms

$$
\left(P \operatorname{gr}_{l}^{M} \operatorname{gr}_{k}^{W} \operatorname{gr}_{\alpha}^{V} \mathscr{M}, F_{0}\right) \stackrel{\sim}{\rightarrow}\left(\operatorname{gr}_{l+1}^{W} \operatorname{gr}_{k}^{W} \mathcal{O}_{x}\left[*\left(F^{-1}(\infty) \cup D\right)\right]_{\alpha}, F_{\circ}\right)
$$

Proof. Let us recall the local computation of $V_{\circ} \mathscr{M}$ analogous to the one of $V_{\circ} \mathscr{E}$ made above. Denote $\mathscr{D}^{\prime}=\mathbb{C}\left\{x, y, z, t^{\prime}\right\}\left\langle\partial_{x}, \partial_{y}, \partial_{z}, \partial_{t^{\prime}}\right\rangle$. Then, in the local setting of the previous section, $\mathscr{M} \subset \mathbb{C}\left\{x, y, z, t^{\prime}\right\}\left[x^{-1}, y^{-1}, \partial_{t^{\prime}}\right] \cdot \delta\left(t^{\prime}-F^{\prime}\right)$ is equal to the $\mathscr{D}^{\prime}$-submodule generated by $\delta^{\prime}=\frac{1}{y_{1} \cdots y_{q}} \delta\left(t^{\prime}-F^{\prime}\right)$. This generator satisfies

$$
\begin{gathered}
t^{\prime} \delta^{\prime}=x^{m} \delta^{\prime}, \quad \partial_{x_{i}} \delta^{\prime}=-\frac{m_{i}}{x_{i}}\left(\partial_{t^{\prime}} \cdot t^{\prime}\right) \delta^{\prime}, \quad \partial_{y_{j}} \delta^{\prime}=-\frac{1}{y_{j}} \delta^{\prime} \\
t^{\prime}\left(\prod_{i=0}^{p} \partial_{x_{i}}^{m_{2}}\right) \cdot \delta^{\prime}=(-m)^{m}\left(\prod_{i=0}^{p} \prod_{k=0}^{m_{i}-1}\left[t^{\prime} \partial_{t^{\prime}}+\frac{k}{m_{i}}\right]\right) \cdot \delta^{\prime} .
\end{gathered}
$$

Hence $\delta^{\prime}$ has order $<1$ with respect to $V_{\circ} \mathscr{M}$. It can be shown that, for $\alpha<1$,

$$
\begin{aligned}
V_{\alpha} \mathscr{M} & =\sum_{a \in \mathbb{Z}^{p+1}} \mathbb{C}\{x, y, z\}\left[y^{-1}, t^{\prime} \partial_{t^{\prime}}\right] x^{m-a-1} P_{a, \alpha}\left(t^{\prime} \partial_{t^{\prime}}\right) \cdot \delta^{\prime} \\
& =\sum_{a \in \mathbb{Z}^{p+1}} \mathbb{C}\{x, y, z\}\left[y^{-1}, t^{\prime} \partial_{t^{\prime}}\right] x^{-a} P_{a-1, \alpha}\left(t^{\prime} \partial_{t^{\prime}}\right) \cdot\left(t^{\prime} \delta\right) \\
& =\sum_{a \geq\lceil m \alpha \mid} \mathbb{C}\{x, y, z\}\left[y^{-1}, t^{\prime} \partial_{t^{\prime}}\right] x^{-a} P_{a-\mathbb{1}, \alpha}\left(t^{\prime} \partial_{t^{\prime}}\right) \cdot\left(t^{\prime} \delta\right)
\end{aligned}
$$

with $\delta=\delta\left(t^{\prime}-F^{\prime}\right)$. We then get a canonical representative of any local section of $V_{\alpha} \mathscr{M}$ and the proof of the analogue of 4.5 can be done as above. The only difference comes from the shift $(+1)$ for $\alpha=0$ which does not apppear here (in fact a shift $(-1)$ would appear at $\alpha=1$, but it will not matter here).

Now the proof is exactly the same as the one of Proposition 4.14.

In the same way one deduces from this proposition 
(4.20) Corollary. For $\alpha \in\left[0,1\left[\right.\right.$ any local isomorphism of $\mathscr{D}_{\mathscr{X} \text {-modules }}$

$$
P \operatorname{gr}_{l}^{M} \operatorname{gr}_{k}^{W} \operatorname{gr}_{\alpha}^{V} \mathscr{M} \stackrel{\sim}{\rightarrow} \operatorname{gr}_{l+1}^{\mathscr{W}} \operatorname{gr}_{k}^{W} \mathcal{O}_{X}\left[*\left(F^{-1}(\infty) \cup D\right)\right]_{\alpha}
$$

is strictly filtered with respect to the filtrations $F_{0}$.

4.21. Proof of Theorem 4.3. As the isomorphism (4.2) is strict with respect to $W_{\bullet}$ and is compatible with the monodromy, it induces an isomorphism between the corresponding $P \operatorname{gr}_{l}^{M} \mathrm{gr}_{k}^{W}$. From Cor. 4.18 and 4.20 we deduce that this one is strict for $G_{0}$ and $F_{0}$. According to Prop. 4.5-(2), Go (resp. $F_{\bullet}$ ) is compatible with the Lefschetz decomposition, hence we conclude that $\operatorname{gr}_{l}^{M} \operatorname{gr}_{k}^{W}(4.2)$ is strict for $G_{\bullet}$ and $F_{\bullet}$, and so is (4.2). This gives the first part of 4.3 for $\phi_{\tau}^{\text {mod }}$. Analogous arguments give it for $\psi_{\tau, 1}^{\text {mod }}$.

From the definition of $\kappa_{+} \mathscr{E}^{-\tau}$ and its filtration $G_{\bullet}$, we have an exact sequence of filtered holonomic $\mathscr{D}_{\mathscr{X}}[\tau]\left\langle\partial_{\tau}\right\rangle$-modules

$$
0 \rightarrow\left(\kappa_{+} \mathscr{E}^{-\tau f}, G_{\bullet}\right) \stackrel{\tau}{\rightarrow}\left(\kappa_{+} \mathscr{E}^{-\tau f}, G_{\circ}[-1]\right) \rightarrow\left(\kappa_{+} \mathcal{O}_{U}, F_{\circ}[-1]\right) \rightarrow 0
$$

which induces the exact sequence of the theorem after taking the graduation by the $V$-filtration. Let us show that it is strict with respect to the Hodge filtrations:

In an analytic neighbourhood of a point of $X=\mathscr{X}-F^{-1}(\infty)$, an easy computation shows that $\phi_{\tau .1}^{\text {mod }}\left(\kappa_{+} \mathscr{E}^{-\tau f}\right)=0$ and the statement is clear. In an analytic neighbourhood of a point of $F^{-1}(\infty)$, the strictness follows from the local computation of Lemma 4.11.

From the first part and Cor. 1.13 it follows that this sequence is isomorphic (with filtrations and rational structure) to the exact sequence (2.22.1) in [24], so is an exact sequence of mixed Hodge modules.

\section{§5. Hodge Properties of the Gauss-Manin System and Its Fourier Transform}

We keep assumptions and notation of $\S 4$. We will now consider Hodge properties of the nearby or vanishing cycles of the Fourier transform $\widehat{\mathbb{R} f_{*}{ }^{p} \mathbf{Q}_{U}}$ at $\tau=0$. Remark first that, since the functors ${ }^{p} \phi_{\tau},{ }^{p} \psi_{\tau, 1}$ and $\longrightarrow$ preserve perversity, the cohomology spaces $H^{i p} \phi_{\tau}\left(\widehat{\mathbb{R} f_{*}^{p} \mathbb{Q}_{U}}\right)$ are equal to ${ }^{p} \phi_{\tau} \widehat{\boldsymbol{R}^{i} f_{*}^{p} \mathbf{Q}_{U}}$ (and the same equality for ${ }^{p} \psi_{\tau, 1}$ ), where ${ }^{p} \mathbb{R}^{i} f_{*}$ denotes the $i$ th perverse cohomology sheaf of $\mathbb{R} f_{*}$. 
We hence have on these spaces a filtration $W_{\bullet}$ :

$$
\begin{aligned}
W_{\bullet} H^{i p} \phi_{\tau}\left(\widehat{\boldsymbol{R} f_{*}{ }^{p} \mathbf{Q}_{U}}\right) & =\operatorname{image}\left[{ }^{p} \phi_{\tau}\left({ }^{p} \boldsymbol{R}^{i} F_{*} W_{\bullet}\left(\boldsymbol{R} j_{*}{ }^{p} \mathbf{Q}_{U}\right)\right) \widehat{\rightarrow}{ }^{p} \phi_{\tau} \widehat{R^{i} f_{*}{ }^{p} \mathbb{Q}_{U}}\right] \\
& ={ }^{p} \phi_{\tau}\left(W_{\circ} \widehat{\boldsymbol{R}^{i} f_{*}{ }^{p} \mathbf{Q}_{U}}\right)
\end{aligned}
$$

where $W_{\bullet}{ }^{p} \boldsymbol{R}^{i} f_{*}{ }^{p} \mathbb{Q}_{U}=$ image $\left[{ }^{p} \mathbb{R}^{i} F_{*} W_{\circ}\left(\boldsymbol{R}_{*}{ }_{*}{ }^{p} \mathbb{Q}_{U}\right) \rightarrow{ }^{p} \boldsymbol{R}^{i} f_{*}{ }^{p} \mathbf{Q}_{U}\right]$ and the image is taken in the perverse sense. The same description holds for ${ }^{p} \psi_{\tau, 1}$. This filtration is identified with the one coming from ${ }^{p} \boldsymbol{R}^{i} \check{p}_{*}\left({ }^{p} \phi_{\tau} \mathscr{F}_{F}\left(\mathbb{R}_{j_{*}}{ }^{p} \mathbf{Q}_{U}\right), W_{\mathrm{o}}\right)$ as follows from Remark 2.3.4.

(5.1) Proposition. On $H^{i p} \phi_{\tau}\left(\widehat{\mathbb{R} f_{*}^{p} \mathbf{Q}_{U}}\right)$ and $H^{i p} \psi_{\tau, 1}\left(\widehat{\mathbb{R} f_{*}^{p} \mathbf{Q}_{U}}\right)$ the monodromy filtration of the nilpotent endomorphism $N$ relative to $W_{\bullet}$ exists.

Proof. According to Th. 2.2 and Cor. 1.13 we have $H^{i p} \phi_{\tau}\left(\widehat{R f_{*}{ }^{p} \mathbf{Q}_{U}}\right)=$ ${ }^{p} \psi_{1 / t}{ }^{p} R^{i} f_{*}{ }^{p} \mathbf{Q}_{U}$ and the assertion for ${ }^{p} \phi_{\tau}$ is a consequence of [26]. For ${ }^{p} \psi_{\tau, 1}$ one may use $[24, \S 2 \mathrm{e}]$.

The spaces $H^{i p} \phi_{\tau}\left(\widehat{\boldsymbol{R} f_{*}{ }^{P} \boldsymbol{C}_{U}}\right)$ and $H^{i p} \psi_{\tau, 1}\left(\widehat{\boldsymbol{R} f_{*}{ }^{P} \boldsymbol{C}_{U}}\right)$ come equipped naturally with an increasing filtration $G_{\circ}$ : the filtration $G_{\circ} \phi_{\tau}^{\bmod }\left(\kappa_{+} \mathscr{E}^{-\tau f}\right)$ introduced previously can be used to filter the complex ${ }^{p} \mathrm{DR} \phi_{\tau}^{\bmod }\left(\kappa_{+} \mathscr{E}^{-\tau f}\right)$; the $i$ th term of the complex $G_{\bullet}{ }^{p} \mathrm{DR} \phi_{\tau}^{\bmod }\left(\kappa_{+} \mathscr{E}^{-\tau f}\right)$ is

$$
\Omega_{\mathscr{X}}^{i+n+1} \otimes G_{i+\bullet} \phi_{\tau}^{\bmod }\left(\kappa_{+} \mathscr{E}^{-\tau f}\right)
$$

We hence get a filtration on the hypercohomology of this complex in a natural way. The same construction holds for ${ }^{p} \psi_{\tau, 1}$.

\section{Remarks.}

1. The filtration $G$ o could depend on the choice of a compactification $\mathscr{X}$ of $f$ such that $\mathscr{X}-U$ is a divisor with normal crossings in $\mathscr{X}$. It is a consequence of the theorem below that it does not.

2. By the very construction of $G_{\bullet} \phi_{\tau}^{\bmod }\left(\kappa_{+} \mathscr{E}^{-\tau f}\right)$, the filtration $G_{\bullet} H^{i p} \phi_{\tau}$ $\left(\widehat{R f_{*}{ }^{p} C_{U}}\right)$ is compatible with the decomposition ${ }^{p} \phi_{\tau}=\oplus_{\lambda}{ }^{p} \phi_{\tau, \lambda}$, in other words it is invariant by the action of the semi-simple part of the monodromy. Moreover, the nilpotent endomorphism $N$ shifts it by one. 
From $[24, \S 2 . c]$ and Theorem 4.3 we get, after a Tate twist by $n$ with $\operatorname{dim} U=n+1$ :

(5.3) Theorem. For all $i \in \mathbf{Z}$ the objects

$$
\begin{aligned}
& {\left[H^{i p} \phi_{\tau}\left(\widehat{\mathbb{R} f_{*}{ }^{p} \mathbf{C}_{U}}\right), G_{\bullet}, M_{\bullet}(N, W)[i-n] \bullet, H^{i p} \phi_{\tau}\left(\widehat{R f_{*}{ }^{p} \mathbf{Q}_{U}}\right)(n)\right]} \\
& {\left[H^{i p} \psi_{\tau, 1}\left(\widehat{\boldsymbol{R} f_{*}{ }^{p} \mathbf{C}_{U}}\right), G_{\bullet}, M_{\bullet}(N, W)[i-(n+1)]_{\bullet}, H^{i p} \psi_{\tau, 1}\left(\widehat{\boldsymbol{R} f_{*}{ }^{p} \mathbf{Q}_{U}}\right)(n+1)\right]}
\end{aligned}
$$

are mixed Hodge structures. Moreover the filtration $W_{\bullet}$ is a filtration by mixed Hodge substructures.

Remark. Since the Hodge filtration is usually decreasing, we use the convention $G^{\bullet}=G_{-}$. to obtain a decreasing filtration from an increasing one. The notation $(k)$ corresponds to the Tate twist $(2 i \pi)^{k}$. More precisely, the first object is isomorphic to the Steenbrink-Zucker limit ${ }^{p} \psi_{1 / t}{ }^{p} \boldsymbol{R}^{i} f_{*}{ }^{p} \mathbf{Q}_{U}$ after a twist by $n$.

The spectrum of $f$ at infinity. Following J. Steenbrink [25], we define, for a finite dimensional vector space $H=\oplus_{\lambda} H_{\lambda}$ (where the direct sum is indexed by the roots of 1) equipped with an increasing filtration $F_{\bullet} H=\oplus_{\lambda} F_{\bullet} H_{\lambda}$, spectral polynomials in $\mathbf{Q}[S]$

$$
\begin{aligned}
& \operatorname{SP}_{\psi}\left(H, F_{\bullet} ; S\right) \stackrel{\text { def }}{=} \prod_{p \in \mathbb{Z}} \prod_{\alpha \in[0,1[}(S+\alpha+p)^{h_{\exp 2 \imath \pi \alpha, p}} \\
& \operatorname{SP}_{\phi}\left(H, F_{\bullet} ; S\right) \stackrel{\text { def }}{=} \prod_{p \in \mathbf{Z}} \prod_{\alpha \in] 0,1]}(S+\alpha+p)^{h_{\exp 2 \imath \pi \alpha, p}}
\end{aligned}
$$

with $h_{\lambda, p}=\operatorname{dim} \operatorname{gr}_{p}^{F} H_{\lambda}$. The polynomial $\mathrm{SP}_{\phi}$ is obtained from $\mathrm{SP}_{\psi}$ by replacing the terms $(S+p)$ with $(S+p+1)$ without changing their exponents.

The spectral rational fraction of $f$ at infinity is obtained by using the Hodge filtration on the nearby cycles at infinity $\boldsymbol{H}^{i}\left(F^{-1}(\infty),{ }^{p} \psi_{1 / f}{ }^{p} \mathbf{C}_{U}\right)=H^{i p} \psi_{1 / t}\left(\boldsymbol{R} f_{*}{ }^{p} \mathbf{C}_{U}\right)$ as defined by J. Steenbrink and S. Zucker [26], F. Elzein [8] or M. Saito [24]:

$$
\mathrm{SP}_{\star}\left({ }^{p} \psi_{1 / t}\left(\boldsymbol{R} f_{*}{ }^{p} \mathrm{C}_{U}\right) ; S\right) \stackrel{\text { def }}{=} \prod_{i} \operatorname{SP}_{\star}\left(H^{i p} \psi_{1 / t}\left(\boldsymbol{R} f_{*}^{p} \mathbf{C}_{U}\right), F_{\bullet} ; S\right)^{(-1)^{1}} \in \mathbf{Q}(S)
$$

with $\star=\psi$ or $\star=\phi$. 
We define in an analogous way $\operatorname{SP}_{t}\left({ }^{p} \phi_{\tau}\left(\widehat{\mathbb{R} f_{*}^{p} \mathbb{C}_{U}}\right) ; S\right)$ by using the filtration $G_{\text {o }}$.

(5.4) Corollary. For $\star=\psi$ or $\star=\phi$ we have

$$
\mathrm{SP}_{\hbar}\left({ }^{p} \psi_{1 / t}\left(\mathbb{R} f_{*}{ }^{p} \mathbb{C}_{U}\right) ; S\right)=\operatorname{SP}_{\star}\left({ }^{p} \phi_{\tau}\left(\widehat{R f_{*}^{P} \mathbb{C}_{U}}\right) ; S\right) .
$$

5.5. Remark. In fact, the spectral fraction of $f$ at infinity following [25] is equal to the rational fraction $\operatorname{SP}_{\phi}\left({ }^{p} \psi_{1 / t}\left(\mathbb{R} f_{*}{ }^{p} \mathbb{C}_{U}\right) ; S-1\right)$ as defined above. Indeed, consider on the space $\mathbb{H}^{k}\left(F^{-1}(\infty), \psi_{1 / f}\left(\mathbb{R} j_{*} \mathbb{C}_{U}\right)\right.$ ) (i.e. the limit of $H^{k}\left(f^{-1}(t), \mathbb{C}\right)$ when $\left.t \rightarrow \infty\right)$ the Hodge filtration $F_{H}^{\circ}$ as constructed in [26] or [24], and the monodromy $T_{\infty}$ (see $\S 1.9$ ). For $\left.\left.\beta \in\right]-1,0\right]$ such that $\exp (-2 i \pi \beta)$ is an eigenvalue of $T_{\infty}$, let $v_{\beta+p}$ be the dimension of $\operatorname{gr}_{F_{H}}^{p}$ of the corresponding generalized eigenspace and put $\operatorname{SP}_{k}(S)=\prod_{p} \prod_{\beta \in]-1,0]}(S+$ $\beta+n-p)^{v_{\beta}+p}$. The spectral rational fraction constructed in [25] is then

$$
\mathrm{SP}(S)=\prod_{k} \mathrm{SP}_{k}(S)^{(-1)^{n-k}}
$$

Let us show that $\operatorname{SP}(S)=\operatorname{SP}_{\phi}\left({ }^{p} \psi_{1 / t}\left(\mathbb{R} f_{*}{ }^{p} \mathbb{C}_{U}\right) ; S-1\right)$. We have

$$
\mathbb{H}^{k}\left(F^{-1}(\infty), \psi_{1 / f}\left(\mathbb{R} j_{*} \mathbb{C}_{U}\right)\right)=\mathbb{H}^{k-n}\left(F^{-1}(\infty),{ }^{p} \psi_{1 / f}\left(\mathbb{R} j_{*}{ }^{p} \mathbb{C}_{U}\right)\right)
$$

and the mixed Hodge structure with filtration $F_{\circ}$ that we use differs from $F_{H}^{\circ}$ by a Tate twist $(n)$. Hence we have

$$
F_{H}^{p} H^{k}\left(F^{-1}(\infty), \psi_{1 / f}\left(\mathbb{R} j_{*} \mathbb{C}_{U}\right)\right)=F_{n-p} H^{k-n}\left(F^{-1}(\infty),{ }^{p} \psi_{1 / f}\left(\mathbb{R}_{*}{ }_{*}^{p} \mathbb{C}_{U}\right)\right)
$$

To define $\operatorname{SP}_{\phi}\left({ }^{p} \psi_{1 / t}\left(\mathbb{R} f_{*}{ }^{P} \mathbb{C}_{U}\right) ; S\right)$ we use $\left.\left.\alpha \in\right] 0,1\right]$ such that $\exp (2 i \pi \alpha)$ is an eigenvalue of $T_{\infty}^{-1}$ and this explains the shift by 1 .

\section{Appendix: Convention and Notation}

We refer to [2] or to chapter 10 in [11] for general results on perverse sheaves.

\section{A.1. Filtrations}

To an increasing filtration $F_{0}$ we associate a decreasing filtration

$$
F^{\circ} \stackrel{\text { def }}{=} F_{-\circ}
$$


The shift is analogous to the one defined by M. Saito, namely

$$
\begin{gathered}
F_{\bullet}[k]=F_{\bullet-k} \\
W^{\bullet}[k]=F^{\bullet+k} .
\end{gathered}
$$

\section{A.2. Perverse Notation for Constructible Sheaves}

Perversity, cohomology and duality. We denote $\operatorname{Perv}\left(\mathbf{Q}_{X}\right)$ the abelian category of $\mathbb{Q}$-perverse sheaves on the manifold $X$. Let $f: X \rightarrow Y$ be a morphism between complex analytic manifolds. Put $\delta_{f}=\operatorname{dim} X-\operatorname{dim} Y$ and ${ }^{p} \mathbf{Q}_{X}$ $=\mathbb{Q}_{X}[\operatorname{dim} X]$. This is a perverse sheaf. Let $\boldsymbol{D}_{X}$ be the Verdier duality functor on the category $D_{c}^{b}\left(\mathbf{Q}_{X}\right)$. We have

$$
\mathbb{D}^{p} \mathbf{Q}_{X} \stackrel{\text { def }}{=} \mathbb{R} \mathscr{H}_{o m} \mathbf{Q}_{X}\left({ }^{p} \mathbf{Q}_{X}, \mathbf{Q}_{X}[2 \operatorname{dim} X]\right) \simeq{ }^{p} \mathbf{Q}_{X}
$$

If $\mathscr{F}$ is an object of $D_{c}^{b}\left(\mathbb{Q}_{X}\right)$, we denote $\mathscr{H}^{i} \mathscr{F}$ the $j$ th cohomology group of $\mathscr{F}$ (this is a sheaf on $Y$ ) and ${ }^{{ }^{2}} \mathscr{H}^{j} \mathscr{F}$ the $j$ th perverse cohomology group (this is a perverse sheaf on $Y$, hence a complex in general). We have

$$
D_{X}{ }^{{ }^{p}} \mathscr{H}^{j} \mathscr{F}={ }^{p} \mathscr{H}^{j} \mathbb{D}_{X} \mathscr{F} .
$$

Inverse image. The inverse image is modified as follows:

$$
p f^{-1}=f^{-1}\left[\delta_{f}\right]
$$

If $f$ is smooth or if $f$ is a closed immersion we have ${ }^{p} f^{-1}{ }^{p} \mathbb{Q}_{Y}={ }^{p} \mathbb{Q}_{X}$. We also put

$$
{ }^{p} f^{!}=f^{!}\left[-\delta_{f}\right]=D_{X}^{p} f^{-1} D_{Y} .
$$

If $f$ is smooth, we have ${ }^{p} f^{!}={ }^{p} f^{-1}$.

Direct image. The direct images $\mathbb{R} f_{!}$et $\mathbb{R} f_{*}$ are unchanged. We put

$$
\boldsymbol{R}^{j} f_{\star}=\mathscr{H}^{i} \mathbb{R} f_{\star} \text { et }{ }^{p} \mathbb{R}^{j} f_{\star}={ }^{p} \mathscr{H}^{j} \mathbb{R} f_{\star}
$$

with $\star * *$ or $\star=$ !.

Nearby and vanishing cycles. Let $f: X \rightarrow \mathbf{C}$. The nearby and vanishing cycles functors are modified in the following way: 


$$
{ }^{p} \psi_{f} \mathscr{F}=\psi_{f} \mathscr{F}[-1] \text { and }{ }^{p} \phi_{f} \mathscr{F}=\phi_{f} \mathscr{F}[-1]
$$

Compatibilities and exact sequences. We have

$$
\begin{aligned}
& { }^{p} f^{!}=D_{X}^{p} f^{-1} D_{Y} \\
& p(f \circ g)^{-1}=p^{-1} p f^{-1} \\
& { }^{p}(f \circ g)^{!}={ }^{p} g^{! p} f^{!} \\
& \boldsymbol{R} f_{!}=\boldsymbol{D}_{\mathbf{Y}} \boldsymbol{R} f_{*} \boldsymbol{D}_{\boldsymbol{X}} \\
& { }^{p} \boldsymbol{R}^{j} f_{1}=\boldsymbol{D}_{Y}{ }^{p} \boldsymbol{R}^{j} f_{*} \boldsymbol{D}_{X} \\
& \boldsymbol{R}(f \circ g)_{\star}=\mathbb{R} f_{\star} \boldsymbol{R} g_{\star} \star=* \text { or ! } \\
& \boldsymbol{D}_{f^{-1}(0)}{ }^{p} \psi_{f}={ }^{p} \psi_{f} \boldsymbol{D}_{X} \quad \text { with } f: X \rightarrow \mathbb{C} \text { smooth } \\
& D_{f^{-1}(0)}^{p} \phi_{f}={ }^{p} \phi_{f} D_{X} \quad \text { idem } \\
& { }^{p} \psi_{f}{ }^{p} \mathscr{H}^{j}={ }^{p} \mathscr{H}^{j p} \psi_{f} \quad \text { idem } \\
& { }^{p} \phi_{f}{ }^{p} \mathscr{H}^{j}={ }^{p} \mathscr{H}^{j p} \phi_{f} \quad \text { idem } \\
& \boldsymbol{R} g_{*}^{p} \psi_{g \circ f}={ }^{p} \psi_{f} \boldsymbol{R g}_{*} \quad X \stackrel{g}{\rightarrow} \underset{Y}{\rightarrow} \mathrm{C}, \quad g \text { proper } \\
& \boldsymbol{R} g_{*}^{p} \psi_{g_{\circ f}}={ }^{p} \psi_{f} \boldsymbol{R} g_{*} \quad \text { idem. }
\end{aligned}
$$

Triangles (with $i: f^{-1}(0) \hookrightarrow X$ ):

$$
\begin{aligned}
& p_{i}^{-1} \longrightarrow{ }^{p} \psi_{f} \stackrel{\text { can }}{\longrightarrow}{ }^{p} \phi_{f} \stackrel{+1}{\longrightarrow} \\
& { }^{p} \phi_{f} \stackrel{\operatorname{var}}{\longrightarrow}{ }^{p} \psi_{f} \longrightarrow{ }^{p} ! \stackrel{+1}{\longrightarrow}
\end{aligned}
$$

If $i: Y \hookrightarrow X$ is a closed immersion and $j: X-Y \hookrightarrow X$ is the complementary open immersion (we then have $j^{-1}=j^{-1}=p_{j} !=j^{!}$), we have triangles

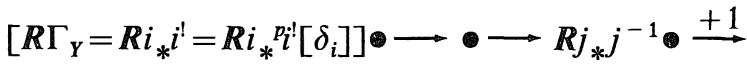

$$
\begin{aligned}
& \boldsymbol{R} j_{!} j^{\prime} \longrightarrow \longrightarrow\left[\boldsymbol{R} i_{*} i^{-1}=R i_{*} i^{p^{-1}}\left[-\delta_{i}\right]\right] \bullet \stackrel{+1}{\longrightarrow}
\end{aligned}
$$

If moreover $Y$ has codimension one in $X$, we have triangles 


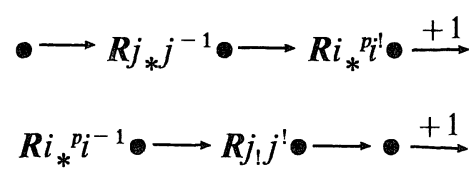

Adjunction:

$$
\begin{aligned}
& \boldsymbol{R} f_{!}\left(\mathscr{F} \otimes f^{-1} \mathscr{G}\right)=\boldsymbol{R} f_{!} \mathscr{F} \otimes \mathscr{G} \\
& \boldsymbol{R} f_{!}\left(\mathscr{F} \otimes f^{-1} \mathscr{G}\right)=\boldsymbol{R} f_{!} \mathscr{F} \otimes \mathscr{G}\left[\delta_{f}\right] .
\end{aligned}
$$

\section{A.3. $\mathscr{D}$-modules}

Here, the category is either the algebraic category or the analytic category, and the objects correspond each other with the functor "an", e.g. $\mathcal{O}_{X}$ and $\mathcal{O}_{X}^{\text {an }}$.

de Rham. We have $\boldsymbol{R} \mathscr{H}_{\circ m_{\mathscr{D}_{X}}}\left(\mathcal{O}_{X}, \mathscr{D}_{X}\right)=\omega_{X}[-\operatorname{dim} X]$. We put

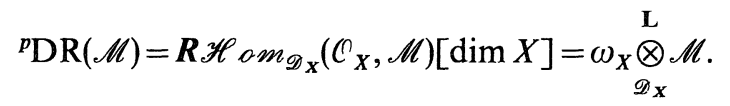

We define in the same way the relative de Rham complex for a projection.

Duality. The duality functor (denoted $D$ or $D_{X}$ ) is centered in such a way that it preserves holonomic modules.

Direct image. We put

$$
f_{+} \mathscr{M}=\boldsymbol{R} f_{*}\left(\mathscr{D}_{\mathbf{Y} \leftarrow X}^{\stackrel{\mathbf{L}}{\bigotimes} \mathscr{D}_{X} \mathscr{M}}\right) .
$$

Notice that $\mathscr{D}_{\mathrm{pt} \leftarrow X}=\omega_{X}$, and consequently for $f: X \rightarrow$ pt we have $f_{+} \mathscr{M}$ $=\boldsymbol{R} \Gamma\left(X,{ }^{p} \mathrm{DR} \mathscr{M}\right)$. In the case of a projection $f: X \times Y \rightarrow Y$ we have $f_{+} \mathscr{M}=\boldsymbol{R} f_{*}{ }^{p} \mathrm{DR} \boldsymbol{f}_{\boldsymbol{f}} \mathscr{M}$

We define

$$
f_{\dagger} \mathscr{M}=D_{Y} f_{+} D_{X} \mathscr{M}
$$

For $f$ proper we have $f_{+}=f_{+}$since in this case $f_{+} D=D f_{+}$.

Inverse image. We put $f^{+} \mathscr{N}=\mathscr{D}_{X \rightarrow \mathbf{Y}} \underset{f^{-1} \mathscr{D}_{\mathbf{X}}}{\stackrel{\mathbf{L}}{\otimes}} f^{-1} \mathscr{N}$ and $f^{\dagger} \mathscr{N}$ 
$=D_{X} f^{+} D_{Y} \mathscr{N}$ with $\mathscr{D}_{X \rightarrow Y}=\mathcal{O}_{X} \otimes_{f^{-1} \mathcal{O}_{Y}} f^{-1} \mathscr{D}_{Y}$, so that as a $\mathcal{O}_{X}$-module, $f^{+}$ corresponds to $\mathbb{L} f^{*}$ (i.e. $\mathcal{O}_{\boldsymbol{X}} \underset{f^{-1} \mathcal{O}_{Y}}{\otimes}$ ). If $f$ is smooth we have $f^{+}=f^{\dagger}$.

Moderate nearby and vanishing cycles. We have triangles (for $f: X \rightarrow \mathbb{C}$ smooth)

$$
\begin{gathered}
i^{\dagger} \longrightarrow \psi_{f}^{\mathrm{mod}} \longrightarrow \phi_{f}^{\mathrm{mod}} \stackrel{+1}{\longrightarrow} \\
\phi_{f}^{\mathrm{mod}} \longrightarrow \psi_{f}^{\mathrm{mod}} \longrightarrow i^{+} \stackrel{+1}{\longrightarrow} .
\end{gathered}
$$

Compatibilities. These are the same as for sheaves, via the following correspondence:

$$
\begin{array}{lll}
f_{+} \leftrightarrow \mathbb{R} f_{*}, & f_{\dagger} \leftrightarrow \mathbb{R} f_{!}, & D \leftrightarrow \mathbb{D} \\
f^{+} \leftrightarrow p^{p} ! & f^{\dagger} \leftrightarrow{ }^{p} f^{-1}, & \psi_{f}^{\bmod }\left(\phi_{f}^{\bmod }\right) \leftrightarrow{ }^{p} \psi_{f}\left({ }^{p} \phi_{f}\right) .
\end{array}
$$

The correpondence is given by ${ }^{p} \mathbb{D}$.

Adjunction ( $f$ analytic and proper or algebraic):

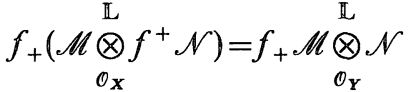

$$
\begin{aligned}
& \mathbb{L} \quad \mathbb{L} \\
& f_{\dagger}\left(\mathscr{M} \underset{\mathcal{O}_{\mathbf{X}}}{\otimes} f^{\dagger} \mathcal{N}\right)=f_{\dagger} \mathscr{M} \underset{\mathcal{O}_{\mathbf{Y}}}{\otimes} \mathscr{N} .
\end{aligned}
$$

\section{References}

[1] Abdel-Gadir, B., Applications of Grauert-Remmert $A$ and $B$ theorems and On the Fourier analysis of holonomic $\mathscr{D}$-modules, preprints, Grenoble 1997.

[2] Beilinson, A. A., Bernstein, J. and Deligne, P., Faisceaux pervers, in Analyse et topologie sur les espaces singuliers, Astérisque, $\mathbb{1 0 0}$ (1982), 7-171.

[3] Björk, J. E., Analytic $\mathscr{D}$-modules and applications, Kluwer Academic Publisher, Dordrecht, 1993.

[4] Brylinski, J. L., Transformations canoniques, dualité projective, théorie de Lefschetz, transformation de Fourier et sommes trigonométriques, in Géométrie et analyse microlocales, Astérisque, $140-141$ (1986), 3-134.

[5] Daia, L., La transformation de Fourier pour les $\mathscr{I}$-modules, Thèse univ. Grenoble (1995).

[6] Deligne, P., Théorie de Hodge II, Publ. Math. IHES, 40 (1971), 5-57.

[7] Théorie de Hodge III, Publ. Math. IHES, 4A (1974), 5-77.

[8] El Zein, F., Théorie de Hodge des cycles évanescents, Ann. Sci. Ec. Norm. Sup., 19 (1986), 107-184. 
[9] Kashiwara, M., The Riemann-Hilbert problem for holonomic systems, Publ. RIMS, Kyoto Univ., 20 (1984), 319-365.

[10] Kashiwara, M. and Schapira, P., Microlocal study of sheaves, Astérisque, 128 (1985).

[11] - Sheaves on Manifolds, Grundlehren Math. Wiss. 292, Springer-Verlag, Berlin, Heidelberg, 1990.

[12] Laumon, G., Transformation de Fourier, constantes d'équations fonctionnelles et conjectures de Weil, Publ. Math. IHES, 65 (1987), 131-210.

[13] Laurent, Y. and Malgrange, B., Cycles proches, spécialisation et $\mathscr{D}$-modules, Ann. Inst. Fourier, 45 (1995), 1353-1405.

[14] Malgrange, B., Equations différentielles à coefficients polynomiaux, Prog. Math., 96, (1991).

[15] Mebkhout, Z., Une équivalence de catégories, Une autre équivalence de catégories, Compositio Math., 51 (1984), 55-62, 63-68.

[16] Le formalisme des six opérations de Grothendieck pour les $\mathscr{D}$-modules cohérents, Hermann, Paris, 1989.

[17] Le théorème de comparaison entre cohomologies de Rham d'une variété algébrique complexe et le théorème d'existence de Riemann, Publ. Math. IHES, 69 (1989), 47-89.

[18] Le théorème de positivité de l'irrégularité pour les $\mathscr{D}_{X}$-modules, in The Grothendieck Festschrift vol. III, Prog. Math., 88 (1990), 83-132.

[19] Mebkhout, Z. and Narváez-Macarro, L., Le théorème de constructibilité de Kashiwara, in Eléments de la théorie des systèmes différentiels vol. 2, les cours du CIMPA, Travaux en cours 46, Hermann, Paris (1993), 47-98.

[20] Mebkhout, Z. and Sabbah, C., D-modules et cycles évanescents, Chapter III §4 in [16].

[21] Sabbah, C., Equations différentielles à points singuliers irréguliers en dimension 2, Ann. Inst. Fourier, 43 (1993), 1619-1688.

[22] - On the comparison theorem for elementary irregular $\mathscr{D}$-modules, Nagoya Math. J., 141 (1996), 107-124.

[23] Saito, M., Modules de Hodge polarisables, Publ. RIMS, Kyoto Univ., 24 (1988), 849-995.

[24] - Mixed Hodge modules, Publ. RIMS, Kyoto Univ., 26 (1990), 221-333.

[25] Steenbrink, J., The spectrum of hypersurface singularities, in Théorie de Hodge, Luminy 1987, Astérisque, 179-180 (1989), 163-184.

[26] Steenbrink, J. and Zucker, S., Variation of mixed Hodge structure I, Invent. Math., 80 (1985), 489-542. 
